\title{
Neuronal Encoding of Visual Motion in Real-Time
}

\author{
Anne-Kathrin Warzecha and Martin Egelhaaf \\ Lehrstuhl für Neurobiologie, Universität Bielefeld, Bielefeld, Germany
}

\section{Contents}

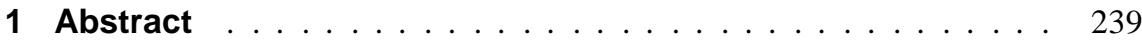

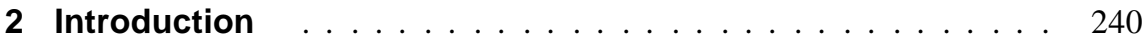

3 The fly as a model system . . . . . . . . . . . . . . . 242

4 How are motion stimuli represented by fly tangential cells? 243

4.1 Representation of the time course of visual motion . . . . . . . . 245

4.2 Variability of motion-induced responses in a spiking neurone . . . 248

4.3 Temporal precision of encoding of visual motion information

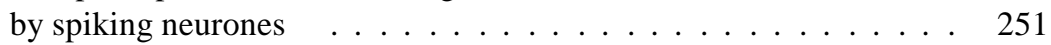

5 What determines the timing of action potentials in fly tangential neurones? . . . . . . . . . . . . . . 253

6 Performance of spiking and graded potential neurones in the encoding of visual motion . . . . . . . . . . . . . . 257

7 Ecological constraints for neuronal representation of motion f. . . . . . . . . . . . . . . . . . . . 261

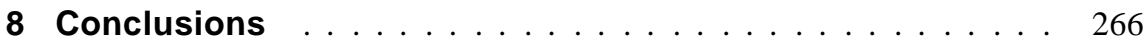

Acknowledgments ..................... 269

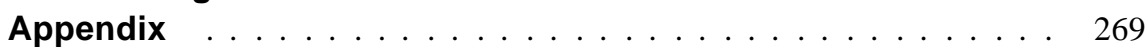

References ........................ 272

\section{Abstract}

Changes in the activity of sensory neurones carry information about a given stimulus. However, neuronal activity changes may also arise from noise sources within or outside the nervous system. Here, the reliability of encoding of visual motion information is analysed in the visual motion pathway of the fly and com- 
pared to the findings obtained in other animal species. Several constraints determine and limit the reliability of encoding of visual motion information: (i) the biophysical mechanisms underlying the generation of action potentials; (ii) the computations performed in the motion vision pathway; and (iii) the dynamical properties of motion stimuli an animal encounters when moving around in its natural environment. The responses of fly motion-sensitive neurones are coupled to visual motion on a timescale of milliseconds up to several tens of milliseconds, depending on the dynamics of the motion stimuli. Only rapid velocity changes lead to a precise time-locking of spikes to the motion stimuli on a millisecond scale. Otherwise, the exact timing of spikes is mainly determined by fast stochastic membrane-potential fluctuations. It is discussed on what timescale behaviourally relevant motion information may be encoded.

\section{Introduction}

We usually take it for granted that we are able to react appropriately in most situations - even when navigating through hectic everyday traffic - and thus to arrive safely at our destination. This capability requires fast and reliable decisions. Such behavioural decisions are inevitably preceded within the organism by a series of neuronal processing steps. First the outside world has to be captured by the sensory system. Then the relevant features of the stimuli have to be extracted in the nervous system from the activity profile of the sensory cells before the motor programs can be initiated to execute the appropriate behavioural reactions. Visual systems of many animals including humans often exploit motion cues to control their path of locomotion. Retinal motion experienced by an organism moving through its environment, the so-called optic flow, provides a wealth of information about the self-motion of the organism and the three-dimensional structure of its surroundings (e.g. Koenderink 1986). The nervous systems of many mobile animals are able to evaluate the optic flow and to exploit this information to mediate appropriate steering manoeuvres (reviews: Miles and Wallman 1993). The activity profile of neurones in the visual motion pathway, thus, provides crucial information about the animal's self-motion and its environment.

Despite the fact that many animals appear to be able to extract without much effort the relevant information about optic flow, the underlying computations are far from being trivial (see also Dahmen et al. this volume ). In particular, the representation of the dynamical features of optic flow in real time is constrained by the underlying neuronal mechanisms:

- The activity level of a motion-sensitive neurone does not unambiguously signal the time course of the retinal velocity within its receptive field. Various combinations of different stimulus parameters such as the velocity of a moving object, its acceleration, texture and contrast, may lead to the same activity level of a given neurone (for review, see Egelhaaf and Borst 1993b). 
- The temporal precision with which sensory stimuli can be encoded by neurones is constrained by the biophysical mechanisms underlying neuronal signal processing.

Many events proceeding in nerve cells are not completely deterministic and thus lead to responses that are unpredictable to a certain degree even if they are elicited by identical stimuli. This uncertainty can have several causes such as the stochastic opening and closing of ion channels (White et al. 2000), or probabilistic transmitter release at chemical synapses (e.g. Allen and Stevens 1994). Moreover, even the sensory input itself may be noisy, as is the case for the visual system due to the stochastic nature of light. Thus, when a given stimulus is repeatedly presented to a neurone, the response exhibits a considerable amount of variability. It has been reported that the variance of the spike count elicited by repeated presentation of a visual stimulus may be as large as the average spike count (e.g. Tolhurst et al. 1983; Vogels et al. 1989; Britten et al. 1993; see also Barberini et al., this volume). Hence, the problem arises how to infer from changes in the neuronal responses whether these changes carry information about the actual stimulus or whether they are just due to noise.

To estimate the response component induced by the stimulus, experimenters usually average over the individual responses to many stimulus presentations. This procedure is intended to eliminate the stochastic component from the neuronal responses. It needs to be mentioned that not all variability observed in responses to identical stimulation is due to stochastic sources. Neuronal variability may also be due to deterministic processes that do not occur time-coupled to the stimulus and thus are not controlled by the experimenter. Because these two sources of neuronal variability cannot easily be separated experimentally, they will be referred to as noise in the following without further distinction. In real life an animal can hardly ever average over the responses to many repetitions of the same situation. It often has only a single and brief chance to react appropriately, for instance in order to evade a predator. A neuronal strategy to increase the signal-to-noise ratio of the neuronal response could be to pool the activity of many equivalent neurones. Indeed, this strategy has been proposed to be exploited by nervous systems (e.g. by neurones in area MT of the monkey, see Zohary et al. 1994; Shadlen et al. 1996). However, it is useful only if the stochastic fluctuations in the signals of the neurones that are being pooled do not covary too much and the pooling neurone itself does not introduce a large amount of noise from its own biophysical machinery. Another strategy to increase the neuronal reliability could be to use the average neuronal activity within a certain time interval. However, by temporally averaging, fast changes in the stimulus cannot be detected in the neuronal activity. Hence, the extent of temporal averaging that helps to increase the signal-to-noise ratio largely depends on the timescale on which the stimulus-induced response component varies.

This paper will focus on the constraints imposed by the neuronal mechanisms underlying visual motion computation on the evaluation of optic flow in real time. An understanding of the real-time performance of motion vision systems 
is essential if we want to understand how optic flow can be exploited by an animal to safely navigate through its environment. In particular, we will discuss the timescale on which visual motion is processed and the temporal precision with which motion stimuli are represented in the visual system. We will concentrate on the motion pathway of the fly and the behavioural responses controlled by this pathway, because a wide range of aspects of visual motion computation have been analysed in this animal in great detail. The capabilities and limitations of the fly visual motion system will be related to other animals and the computational tasks they have to solve.

\section{The fly as a model system}

Flies of the families Muscidae, Calliphoridae and Sarcophagidae have provided us with a well established model system for the analysis of motion information processing because both the behavioural and the neuronal level are well amenable to experimental analysis (for reviews see e.g. Reichardt and Poggio 1976; Egelhaaf et al. 1988; Hausen and Egelhaaf 1989; Bialek and Rieke 1992; Egelhaaf and Borst 1993a; Egelhaaf and Warzecha 1999). Visually guided flight manoeuvres of these insects can be investigated in free flight or with a flight simulator under the well controlled laboratory conditions of tethered flight. Some of these flight manoeuvres are impressively aerobatic and outperform those of most other animals as well as those of any man-made machine. One of the most virtuosic visually controlled manoeuvres of flies is the chasing behaviour during which male flies chase potential mates in order to catch and finally mate with them (Land and Collett 1974; Wagner 1986a). Other well studied behavioural routines that are controlled visually include the detection of objects on the basis of relative motion between the object and its background (Virsik and Reichardt 1976; Reichardt et al. 1983; Heisenberg and Wolf 1984; Egelhaaf et al. 1988; Götz 1991; Egelhaaf and Borst 1993a; Kimmerle et al. 1996; Kimmerle et al. 1997) and the ability to stabilize the flight path against disturbances by compensatory optomotor reactions (Götz 1968; Götz 1975; Reichardt and Poggio 1976; Heisenberg and Wolf 1984; Egelhaaf et al. 1988; Egelhaaf and Borst 1993a; Warzecha and Egelhaaf 1996).

The details of the neuronal circuits controlling components of the visually guided behaviour of the fly are in some instances known down to the level of individual cells (for review see Egelhaaf et al. 1988; Egelhaaf and Borst 1993a). All these neuronal circuits have in common that the retinal images are initially processed in the first and second visual neuropile by successive layers of retinotopically arranged columnar neurones (e.g. Järvilehto et al. 1989; Strausfeld 1989; Laughlin 1994). In the third visual neuropile, the main centre for optic flow analysis, the retinotopic organization of the columnar elements is abandoned. In this brain region, about 40 to 60 so-called tangential cells pool with their extended dendrites the output signals of many excitatory and inhibitory motion-sensitve 
columnar neurones that have opposite preferred directions (see Douglas and Strausfeld, this volume). As a consequence of this input the tangential cells have very large receptive fields (Hausen and Egelhaaf 1989; Egelhaaf and Borst 1993a; Egelhaaf and Warzecha 1999). Owing to this input and to synaptic interactions with other tangential cells they obtain their characteristic sensitivity to optic flow patterns (Hausen 1981; Egelhaaf and Borst 1993a; Egelhaaf and Warzecha 1999; Krapp 1999; Horstmann et al. 2000; see also Dahmen et al., this volume ). The various tangential cells differ in their preferred direction of motion, in their receptive field properties and in their response mode. During visual motion stimulation some of them respond with a sequence of spikes, others with graded changes of their membrane potential and still others mainly in the graded mode, however, with additional spike-like depolarizations (Hengstenberg 1977; Hausen 1982a; Haag et al. 1997; Haag and Borst 1998). The tangential cells can be identified individually in each animal on the basis of their physiological and anatomical properties making them ideal for a quantitative analysis of their performance.

In particular, the spike activity of one of these neurones, the so-called H1cell, can be recorded over extended periods of several hours. Hence, this neurone is well suited for an experimental analysis of the reliability of neural coding and has been used as a model system for this purpose in quite a number of studies (Mastebroek 1974; Gestri et al. 1980; de Ruyter van Steveninck and Bialek 1988; Bialek et al. 1991; de Ruyter van Steveninck and Bialek 1995; Haag and Borst 1997; de Ruyter van Steveninck et al. 1997; Warzecha and Egelhaaf 1997; Warzecha et al. 1998; Warzecha and Egelhaaf 1999).

\section{How are motion stimuli represented by fly tangential cells?}

Fly tangential cells respond directionally selective to visual motion. Spiking tangential cells increase their spike rate above the resting level during motion in their preferred direction and decrease the spike rate during motion in the opposite direction. Since the spontaneous activity of most spiking tangential cells is low, they usually stop firing when the pattern moves in the null direction. Tangential cells that encode their input signals by graded changes in their membrane potential depolarize during preferred direction motion and hyperpolarize during null direction motion (Hausen 1982a; Hengstenberg 1982; Hausen and Egelhaaf 1989). How spiking tangential neurones in the fly's third visual neuropil represent visual motion is shown for one example, the H1-cell, in figure 1.

The fly was stimulated in this example by a grating moving with either a constant velocity or randomly fluctuating velocities. At the onset of constantvelocity motion the spike activity of the H1-neurone increases and, after a transient phase, reaches a more or less constant steady-state level. Even during the steady-state, the interspike intervals are not of constant length but vary considerably. During dynamic stimulation the spike activity fluctuates strongly, following 
to some extent the pattern velocity. When comparing individual response traces elicited by identical motion stimuli, it becomes obvious that the spike activity is not entirely determined by the motion stimulus, but is also driven by some other process, possibly due to noise. Although the overall pattern of spike activity looks quite similar in each of the individual responses, there is some variability in their temporal fine structure across trials (de Ruyter van Steveninck et al. 1997; Warzecha et al. 1998; Warzecha and Egelhaaf 1999). Similar responses can be observed for other spiking tangential cells. Furthermore, tangential cells with graded changes of their membrane potential exhibit similar properties with respect to the motion-induced response component and show variability in the fine structure of their responses across trials (Fig. 6 and Warzecha 1994; Haag and Borst 1997, 1998; Warzecha et al. 1998).

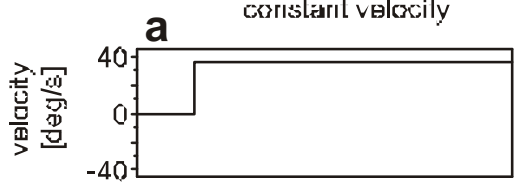

荝

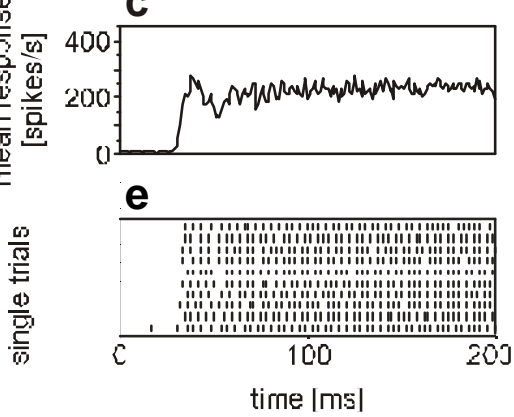

b dyriarric: veloc ly

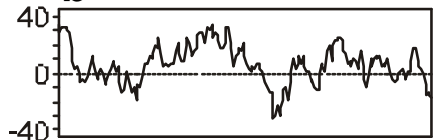

d

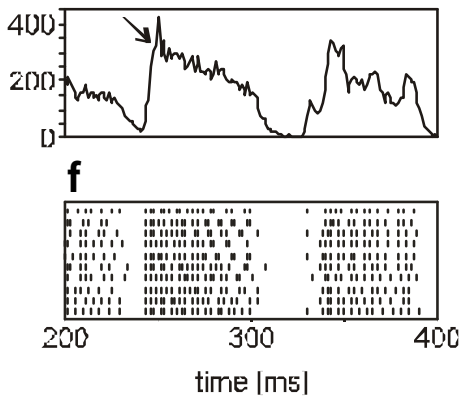

Fig. 1 Responses of the H1-cell to constant and dynamic velocity stimuli. a, b Velocity profile of the stimulus. Squarewave gratings were used as stimuli (wavelength: $18.2^{\circ}$, contrast: 0.99 ) and displayed on a monitor covering the central part of the cell's receptive field (horizontal extent: $91^{\circ}$, vertical extent: $76.6^{\circ}$ ). To obtain the dynamical motion stimuli, white-noise velocity fluctuations were generated according to a Gaussian distribution with a standard deviation of $0.57^{\circ} / \mathrm{ms}$. The resulting velocity trace was lowpass-filtered with a cutoff at $80 \mathrm{~Hz}$ to avoid aliasing due to the frame rate limit of $183 \mathrm{~Hz}$. c, d Spike frequency histogram obtained from the responses of $5 \mathrm{H} 1$-cells to repetitive presentation of the motion traces shown in (a) and (b). For each of the five neurones between 140 and 300 responses were averaged. The responses were shifted by $30 \mathrm{~ms}$ to compensate for the latency between stimulus and response. Temporal resolution: $1 \mathrm{~ms}$. e, f Subsequent sample traces of individual responses to repetitive presentation of the same motion trace plotted underneath each other. Each vertical line denotes the occurrence of a spike. The section of the responses shown in (e) and (f) corresponds to the section of the spike frequency histogram shown in (c) and (d). (a,c,e modified from Warzecha and Egelhaaf 1999) 
To understand what information about the time course of the continually changing retinal input is encoded by fly tangential neurones and how reliably this is accomplished the following topics will be discussed:

- The encoding of dynamic velocity stimuli by the motion-induced response component of the tangential neurones will be analysed.

- The variance between consecutive responses to identical stimuli will be determined in order to find out whether the variability of spike responses is affected by the stimulus dynamics.

- The temporal precision, i.e. the extent to which spikes are time-locked to visual motion, will be analysed by comparing the timing of spikes in consecutive response traces.

\subsection{Representation of the time course of visual motion}

By averaging over many individual responses to the identical motion stimulus we eliminate the noise component and derive the component of the response that is induced by the motion stimulus. For a spiking neurone the motion-induced response component corresponds to the spike frequency histogram. Interestingly, the motion-induced response components of spiking and graded potential neurones elicited by the same stimuli are quite similar. When the fly is stimulated with constant velocity, the motion-induced response is often more or less constant after a brief transient phase (Figs. 1c and e). Sometimes the response decreases slightly over several seconds (Maddess and Laughlin 1985). The response amplitude is modulated only slightly by the spatial structure of the moving pattern. When the velocity fluctuates randomly, the motion-induced response component is strongly modulated, occasionally reaching momentary peak frequencies of more than 300 spikes/s (Fig. 1d). Although the time course of the motion-induced response component is modulated by velocity changes, it is not proportional to the velocity of the stimulus pattern. Particularly pronounced velocity transients in the cell's preferred direction lead to considerably larger responses than expected for a cell which represents the pattern velocity. This is particularly obvious at the onset of the constant velocity stimulus but also during dynamical motion stimulation when the stimulus reverses its direction of motion (see e.g. arrow in Fig. 1d). Indeed, the time course of the motion-induced response of fly tangential neurones does not only depend on pattern velocity but also, in a non-linear way, on acceleration and higher-order temporal derivatives (Egelhaaf and Reichardt 1987; Egelhaaf and Borst 1989). These deviations of the responses from being proportional to pattern velocity are most prominent when the motion stimulus reverses its direction frequently and when the instantaneous velocity becomes large (Egelhaaf and Reichardt 1987, see Fig. 2).

Information about the time course of pattern motion cannot only be derived from the motion-induced response component, as obtained by averaging over many individual response traces, but also in real-time by temporal filtering the 
individual time-dependent responses. The linear filter which leads to an optimal estimation of the instantaneous velocity has been derived both for spiking tangential cells and for tangential cells which encode motion information mainly with graded potentials (Bialek et al. 1991; Haag and Borst 1997). The time course of pattern velocity can be estimated quite well in this way for low frequencies and sufficiently small stimulus amplitudes (Haag and Borst 1997), in accordance with the results displayed in figure 2 . The deviations of the time-dependent responses of tangential cells at high frequencies and large amplitudes from the time course of stimulus velocity are to a large extent the consequence of nonlinearities in the mechanisms underlying motion computation (Egelhaaf and Reichardt 1987; Haag and Borst 1997).

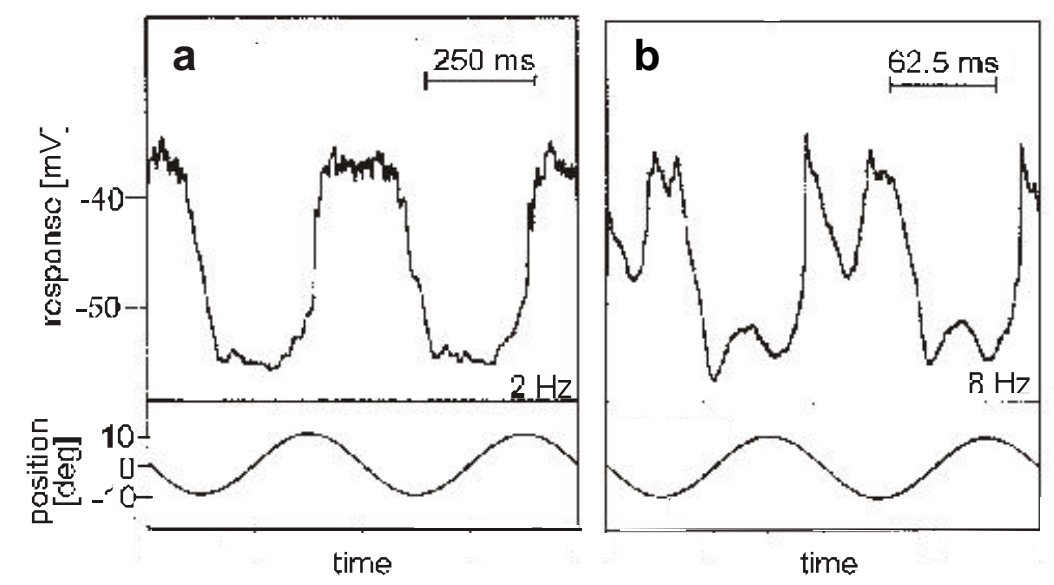

Fig. 2 Dynamical response properties of HS-cells. The cell was stimulated by a grating pattern oscillating sinusoidally with $2 \mathrm{~Hz}$ (a) or $8 \mathrm{~Hz}$ (b) at an amplitude of $\pm 10^{\circ}$ (see bottom traces; note the different time scales). HS-cells signal information about visual motion mainly with graded membrane potential changes that can be superimposed by small spike-like events. At $2 \mathrm{~Hz}$ the response follows the time course of the pattern velocity smoothly and the cell is depolarized during front-to-back motion and hyperpolarized during back-to-front motion; thus the response more or less follows the pattern velocity. At $8 \mathrm{~Hz}$ characteristic distortions become prominent, since hyperpolarizing deflections of the membrane potential occur during front-to-back motion and depolarizing deflections during back-to-front motion. Thus, the response profile is no longer proportional to the time course of pattern velocity if the velocity changes get too transient. Averages from 16 stimulus presentations. (Modified from Egelhaaf and Reichardt 1987).

There are indications that motion-sensitive neurones of other species resemble fly tangential neurones with respect to their representation of the time course of pattern velocity (Movshon et al. 1990; Ibbotson et al. 1994; Lisberger and Movshon 1999). For example, the responses of neurones in area MT of macaque monkeys, which may be involved in the control of pursuit eye movements, do not 
only depend on the velocity but also on the acceleration of pattern motion (Movshon et al. 1990; Lisberger and Movshon 1999) in a way reminiscent of fly tangential neurones.

The peculiar dynamical properties of directionally selective neurones, such as fly tangential cells, can partly be understood as the consequence of the nonlinearities and the time constants that are necessary constituents of any motion detection mechanism (for review see Borst and Egelhaaf 1989; Egelhaaf and Borst 1993b). The time constants involved in motion computation have been reported to cover a range of ten to several tens of milliseconds in a variety of animal species that are phylogenetically rather far apart (arthropods: for review see Egelhaaf and Borst 1993b; monkeys: Mikami et al. 1986). There are three further consequences of the mechanism underlying motion detection which need to be considered here:

- Even the steady-state response amplitude of motion-sensitive neurones increases with increasing velocity only within a limited velocity range (for review, see Buchner 1984; Egelhaaf and Borst 1993b). At a certain velocity the responses reach a maximum and then decrease when the velocity further increases. It should be noted that this kind of velocity dependence is not specific to fly tangential neurones but seems to be a general feature of motionsensitive neurones.

- As a consequence of time-constants inherent to the processes underlying motion computation, the power spectrum of the motion-induced response component in fly tangential neurones obtained from velocity fluctuations containing a wide range of frequencies falls off earlier than that of the motion stimulus (Fig. 3). Through such a lowpass characteristic high-frequency velocity changes are greatly attenuated by the fly motion vision system (Haag and Borst 1997; Warzecha et al. 1998). As might be expected from the time constants described for the processes underlying motion computation in other animal species, this property appears to be a general feature of motion vision systems. For instance, the mean responses of neurones in area MT of the monkey exhibit a similar lowpass characteristic (Bair and Koch 1996).

- The time-constants of the motion detection system in the fly and other insects have been concluded not to be constant, but to adapt to some extent (Maddess and Laughlin 1985; de Ruyter van Steveninck et al. 1986; Borst and Egelhaaf 1987; Harris et al. 1999; see also Maddess this volume). It is assumed that in this way the operating range of the activated neurones is extended so that they are most sensitive under the prevailing stimulus conditions.

In conclusion, the motion-induced response component of fly tangential neurones as well as neurones in other visual systems does not faithfully represent the time course of the retinal velocity. The representation of velocity is highly ambiguous, since the responses do not only depend on pattern velocity but also on the acceleration and higher temporal derivatives of the velocity as well as on the adaptational state of the motion detection system. Moreover, it has long been known that, the responses also depend on the texture and contrast of the stimulus pattern (for review, see e.g. Egelhaaf and Borst 1993b). These characteristic features of 
motion-induced responses need to be taken into account if one wants to understand how optic flow is encoded in real-time, in situations as they are encountered by an animal moving around in its environment.

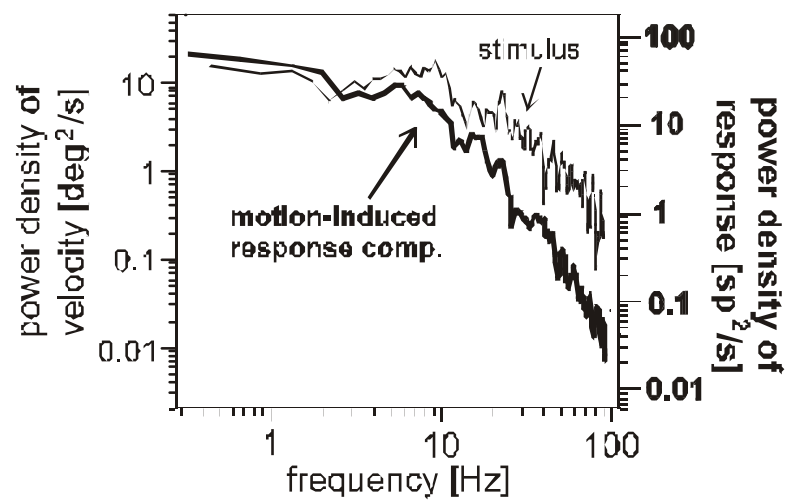

Fig. 3 Frequency response characteristic of the fly motion detection system. The thin line corresponds to the power spectrum of random velocity fluctuations which were used as motion stimuli (left scale). The thick line represents the power spectrum of the stimulus-induced response component of four H1-cells (right scale). The high frequency velocity components in the motion stimulus are attenuated by the motion detection system. For illustration the power spectra were smoothed by a 5 point boxcar filter. (Modified from Warzecha et al. 1998).

\subsection{Variability of motion-induced responses in a spiking neurone}

Obviously, an animal does not have the motion-induced response component at its disposal for controlling behavioural reactions. Instead, the motion-induced response component is contaminated by stochastic fluctuations in the individual responses of a neurone (see Figs. 1 and 6). This stochastic component constrains how reliably motion-induced information can be conveyed by neurones. For motion-sensitive tangential cells of the fly the reliability of coding has been suggested to be especially adapted to dynamical stimuli as they are encountered by an animal in its natural behavioural context (de Ruyter van Steveninck et al. 1997). The variance across trials has been used to quantify how variable individual responses to the same stimulus are within a given time interval relative to the onset of stimulation. Whereas during constant velocity stimulation the variance has been concluded to be in the range of the mean activity, the variance during dynamic velocity stimulation was found to be considerably smaller. On this basis, the $\mathrm{H} 1$-cell has been interpreted to represent dynamic stimuli more reliably than constant ones (de Ruyter van Steveninck et al. 1997). A different conclusion however is drawn from the results that are presented in the following. 


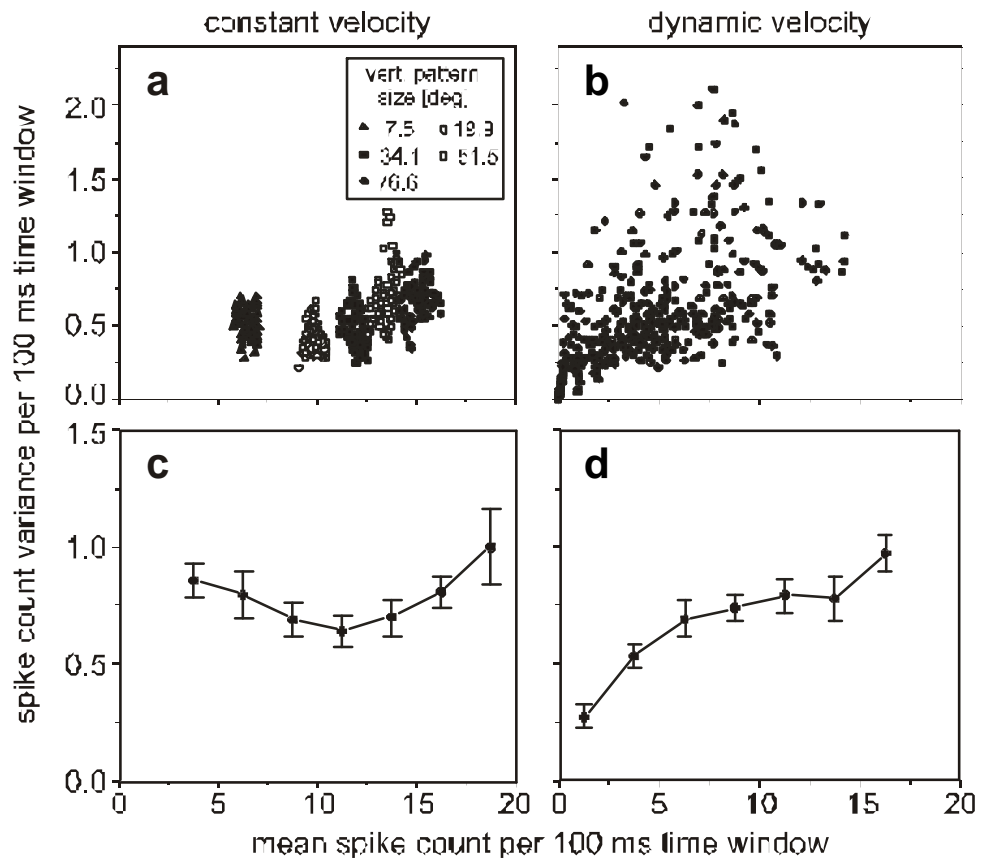

Fig. 4 Variance of the spike count of the H1-cell as a function of the mean count. Spikes were counted in consecutive trials within time windows of $100 \mathrm{~ms}$ time-locked to the onset of motion. Responses were obtained during constant $(\mathbf{a}, \mathbf{c})$ and dynamic $(\mathbf{b}, \mathbf{d})$ velocity stimulation (stimuli as in Fig. 1). The six stimulus conditions (5 constant velocity stimuli and 1 dynamical motion stimulus) were presented in a pseudo-random order before the next sequence started. a, c For constant velocity stimulation different response levels were obtained by presenting 5 stimuli that differed in their vertical extent (different symbols), centred at the cell's receptive field. The motion stimulus lasted for $2.5 \mathrm{~s}$. Mean spike counts and variances were evaluated in 91 consecutive time windows starting $1.5 \mathrm{~s}$ after the motion onset. Consecutive time windows overlapped by $90 \mathrm{~ms}$. b, d Dynamic velocity stimulation with the largest stimulus pattern used in (a) leads to pronounced response modulations (see Fig. 1d), which cover the whole activity range of the H1cell. The motion stimulus lasted for $5 \mathrm{~s}$. Mean spike counts and variances were evaluated in 481 consecutive time windows starting $100 \mathrm{~ms}$ after the motion onset. Consecutive time windows overlapped by $90 \mathrm{~ms}$. a, b Data from one cell. Each symbol indicates the mean and corresponding variance within a given $100 \mathrm{~ms}$ interval time-locked to the onset of motion. c, d Data combined from 8 cells: The mean spike count was subdivided into activity classes with a width of 2.5 spikes per time window. Variances of each cell were averaged, if the corresponding mean spike count fell into the same activity class. It occurred for the analysis done for (c), that the variances of responses elicited by stimuli of different sizes fell into the same activity class and were therefore combined for the mean variance in that activity class. Then the mean variances of up to 8 cells were averaged for each activity class. Only those variances and corresponding activity classes are illustrated to which at least 4 cells contributed. Error bars denote SEMs. For each cell 60 individual responses were evaluated. (c, d modified from Warzecha and Egelhaaf 1999). 
For constant as well as dynamic velocity stimulation the variance of the individual responses across trials is shown in the figure 4 . The mean spike count was determined across trials within time windows time-locked to the onset of motion, and the variance of the spike count within these same time windows was calculated (Warzecha and Egelhaaf 1999). The variances obtained for the two different stimulus dynamics are very similar. The variance does not equal the mean spike count but is markedly smaller than the latter irrespective of stimulus dynamics. Moreover, the variances for the different stimulus dynamics are in the same range. De Ruyter van Steveninck et al. (1997) might have been led to a divergent conclusion because they evaluated the relationship between variance and mean spike count of responses to constant and dynamical motion stimulation in different ways (for details, see Warzecha and Egelhaaf 1999). It should be noted that the response variance across trials is a measure to quantify neuronal variability. Without further qualification, this measure does not allow one to answer the question to what extent information is represented by the exact timing of individual action potentials.

It is interesting to note that in accordance with our data on the fly H1-neurone, there is evidence that in motion-sensitive neurones in area MT the variances of responses to stimuli with different dynamical properties are fairly similar (Buracas et al. 1998). However, in contrast to the fly H1-neurone, in directionally selective neurones in the visual cortex of cats and monkeys (e.g. Britten et al. 1993; Geisler and Albrecht 1997; Buracas et al. 1998; Gershon et al. 1998; see however Gur et al. 1998) the variance was found to be at least as large as the mean spike rate (see also Barberini et al., this volume). The difference between the variability of responses of motion-sensitive cells in flies and "higher" vertebrates most likely does not have its cause in different reliabilities of the spike generating mechanisms. As is suggested by the reliable responses of cortical neurones to current injection (Mainen and Sejnowski 1995; Stevens and Zador 1998), the large variability in cortical neurones may originate from input fluctuations rather than from noise sources intrinsic to the neurones themselves. In general, cortical neurones do not only receive their input signals from peripheral elements along a given sensory pathway but also to a large extent from cortical feedback loops. Therefore, the large variability of responses of cortical neurones might be due to fluctuations of the latter type of input signals, which cannot be expected to timelock to a given sensory stimulus. In contrast to cortical feedback circuitry, fly motion-sensitive neurones are likely to receive their input signals from neurones in the visual motion pathway which reside at the preceding or the same level of motion processing. There is no evidence for recurrent input from subsequent levels of processing. Hence, the different input organization of cortical and fly motion-sensitive neurones may be the reason for their different levels of variability (see also Barberini et al. this volume). 


\subsection{Temporal precision of encoding of visual motion information by spiking neurones}

In order to quantify the temporal precision with which neuronal responses are coupled to the stimulus, we calculated the crosscorrelation between the different spike trains elicited by the same dynamic velocity motion. The power spectrum of the stimulus velocity covered large parts of the dynamic range of motion stimuli that are likely to be encountered by a fly in a natural situation (see Section 6). If there were no jitter in the timing of action potentials across trials, the spikes would be precisely locked to the stimulus on a millisecond timescale and the mean crosscorrelogram (CCG) would be identical to the autocorrelogram of the spike train. It should then exhibit a sharp peak at time zero.
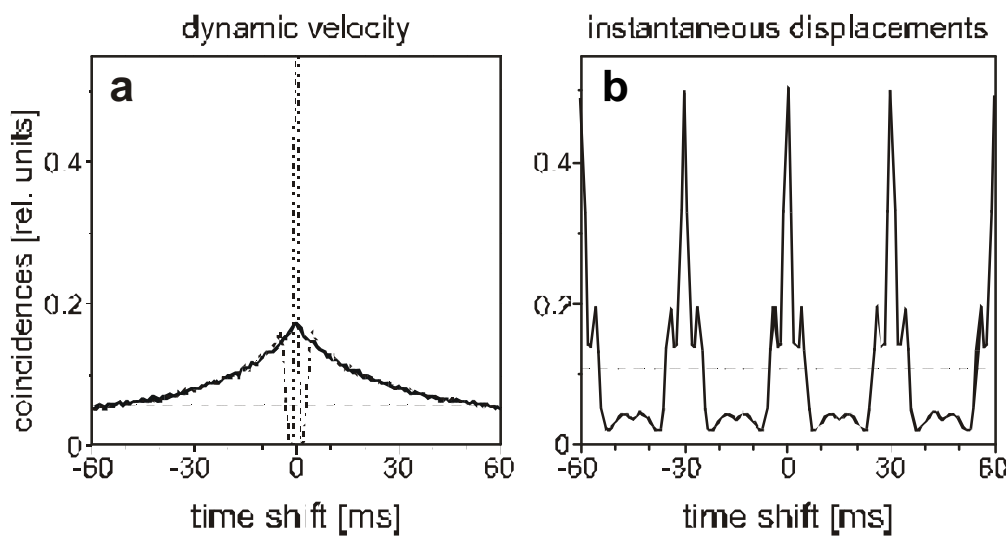

Fig. 5 Time-locking of action potentials to visual stimuli. Average crosscorrelation between pairs of individual spike responses of the H1-cell to identical stimulation. An ordinate value of 1 indicates that individual responses are identical at a temporal resolution of $1 \mathrm{~ms}$. The dashed lines indicate the level of random coincidences expected on the basis of the mean activity. Temporal resolution: 1ms. a Crosscorrelogram (thick line, CCG) and autocorrelogram (dotted line) between 105 pairs of individual spike trains in response to dynamical motion. For the CCG, pairs were chosen pseudorandomly ensuring that every individual response trace was used and that no trace was correlated with itself. The autocorrelogram is shown only partly; at time zero it reaches a value of 1 . Stimulus conditions as in figures $1 \mathrm{~b}$ and $\mathrm{d}$. The time interval evaluated for the correlograms started $100 \mathrm{~ms}$ after the motion onset and lasted for $4.9 \mathrm{~s}$. The mean spike activity within this interval was 57 spikes/s. b CCG between 20 pairs of spike trains in response to instantaneous displacements of a squarewave grating in the cell's preferred direction that occurred every $30 \mathrm{~ms}$. Stimuli were displayed via an LED-array that allowed to present a bright high-contrast wide-field stripe pattern and to displace this pattern within microseconds (mean luminance: $400 \mathrm{~cd} / \mathrm{m} 2$; contrast: 1.0 ; extent of stimulus pattern: horizontal: $252^{\circ}$, vertical $46^{\circ}$; pattern wavelength: $36^{\circ}$; displacement: $2.25^{\circ}$ ). The time interval evaluated for the CCG started $1 \mathrm{~s}$ after the onset of the pattern displacements and lasted for $3 \mathrm{~s}$. The mean spike activity within this interval was 108 spikes/s. (Data shown in b provided by B. Kimmerle). 
In figure 5a an example of a mean $\mathrm{CCG}$ of responses to dynamic velocity motion is shown normalized to the peak amplitude of the corresponding mean autocorrelogram. The CCG is characterized by a rather broad and flat peak around time zero as compared to the width of the mean autocorrelogram. Hence, most action potentials of the H1-neurone are temporally coupled to the transient velocity fluctuations on a timescale of tens of milliseconds but not with a millisecond precision. The temporal precision with which spikes couple to visual motion strongly depends on the dynamics of the motion stimulus. Increasingly less action potentials are time-coupled to the motion stimulus when the velocity changes get smaller and less transient. The CCG between the different spike trains elicited by a constant velocity stimulus is basically flat (for details, see Warzecha et al. 1998).

These conclusions are in accordance with previous findings. The performance in discriminating between brief sections of dynamic motion sequences on the basis of the corresponding spike patterns as well as the reconstruction of pattern velocity from individual spike trains has been concluded to be robust to errors of several milliseconds in spike timing (de Ruyter van Steveninck and Bialek 1988; Bialek et al. 1991).

All these findings are not meant to imply that spikes in motion sensitive neurones of the fly cannot precisely time-lock to visual motion stimuli. CCGs only reflect a kind of average time-locking of spikes to the stimulus and, thus, do not tell us much about neuronal precision during special episodes of the time-dependent spike trains. Precise time-locking of spikes on a millisecond scale may occur when the velocity changes very rapidly. For instance, large instantaneous displacements of a spatially extended, high-contrast pattern in the cell's preferred direction leads to a precise time-locking of action potentials as can be inferred from the pronounced and narrow peaks in the corresponding CCG (Fig. 5b). Even less transient stimuli than instantaneous pattern displacements may lead to a precise time-locking of spikes. For instance, when the pattern suddenly reverses its direction of motion from null-direction to preferred direction motion, the initial spikes may be very precisely timed. This precision is reflected by a small temporal jitter of the first spike at the onset of a period of elevated firing (see arrow in Fig. 1d). The standard deviation of the timing of the first spike can lie below 1-2 ms at such instances for the H1-cell (see also Fig. 2 in de Ruyter van Steveninck et al., this volume). This precision is in accordance with that of neurones in cortical area MT of monkeys (Bair and Koch 1996) as well as of salamander and rabbit retinal ganglion cells (Berry et al. 1997).

It might be desirable to determine the standard deviation of spike jitter not only at the onset of motion transients when no spike has been generated for some time, but also during continuous motion. Although, the spike jitter relative to the time-dependent stimulus can be determined everywhere along a response trace, the result needs to be interpreted with caution. A standard deviation of the spike jitter as small as a few milliseconds indicates precise time-locking to the stimulus only if it is significantly smaller than, roughly speaking, half the interspike interval, which means, for instance, $2.5 \mathrm{~ms}$ for a firing rate of $200 \mathrm{~Hz}$. In this case the 
small spike jitter contingent on the stimulus will also show up in distinct peaks in the spike-frequency histograms, as can be seen, for instance, at the onset of rapid motion (see Fig. 1d of the present account and Fig. 2 in de Ruyter van Steveninck this volume).

In conclusion, spikes can time-lock to visual motion on a wide range of timescales, depending on the dynamics of velocity changes. Which of these timescales is of functional significance for the animal can only be assessed if motion stimuli as they occur in behavioural situations are taken into account (see Section 7).

\section{What determines the timing of action potentials in fly tangential neurones?}

The timing of spikes is mainly determined by the membrane potential and its temporal changes at the spike initiation zone. Therefore we analysed the dynamical properties of the motion-induced and stochastic components of the intracellular membrane potential of tangential neurones. In spiking tangential neurones, like the H1-cell, the postsynaptic potentials cannot easily be derived from intracellular recordings because spikes are superimposed on the postsynaptic potentials. Fortunately there are other tangential neurones such as the HS-cells (Hausen 1982a,b), in which the postsynaptic membrane potential is much less affected by active membrane properties. These cells respond mainly with graded membrane-potential changes which may be superimposed by small spike-like depolarizations. Their receptive fields largely overlap with that of the H1-cell. Since the input organization of these two cell-types is thought to be in principle the same (Hausen 1981), we proceed on the assumption that the graded potential changes recorded in HS-cells can be regarded as a "model" of the synaptically induced membranepotential fluctuations that underly the spike activity in the H1-cell.

White-noise velocity fluctuations were used to determine the dynamic properties of the postsynaptic potentials elicited in one of the HS-cells (for details, see Warzecha et al. 1998). In response to these velocity fluctuations, the membrane potential de- and hyperpolarizes by several millivolts (Fig. 6a). The motioninduced response component obtained from averaging the responses to many presentations of the same motion stimulus (Fig. 6b), is similar to but somewhat smoother than the individual responses. The difference between a single response trace and the motion-induced response component yields the stochastic response component of the respective response trace (Fig. 6a-c). The power spectra of the stochastic and motion-induced response component are shown in figure $6 \mathrm{~d}$. The motion-induced response component contains most power below $20 \mathrm{~Hz}$ although the stimulus contained much higher frequencies. In the low frequency range, the power of the motion-induced response component is larger than that of the stochastic response component. Towards higher frequencies, the power of the motion-induced response component decreases steeply, similar to what has been 
described above for the motion-induced response component of the H1-cell (Fig. 6d). At frequencies above $30-40 \mathrm{~Hz}$, the stochastic response component contains more power than the motion-induced component. Similar results were obtained by Haag and Borst $(1997,1998)$.
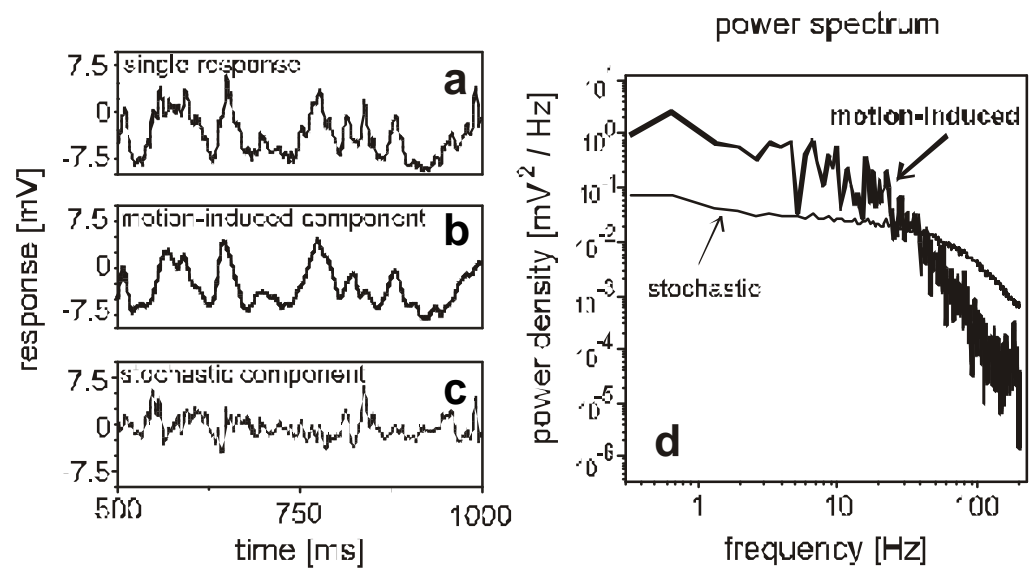

Fig. 6 Dynamic properties of the motion-induced and the stochastic response component. White noise velocity fluctuations were used to determine the dynamic response properties of the postsynaptic potentials elicited in one of the HS-cells, the HSN-cell. The resting potential of the HSN-cell $(-53.4 \mathrm{mV})$ was set to $0 \mathrm{mV}$ for illustration purposes. a Section of a sample record of an individual response trace. $\mathbf{b}$ Motion-induced response component as obtained from averaging 101 responses to the same motion trace. The same time interval relative to motion onset is shown as in (a). c Sample trace of the stochastic response component as obtained from the difference between the motion-induced response shown in (b) and the single response trace shown in (a). d Power spectrum of both the motion-induced response component (thick line) and of the stochastic response component averaged over 101 individual power spectra (thin line). (Modified from Warzecha et al. 1998)

How do these results help us to assess what factors determine the exact timing of action potentials in tangential cells? We have shown that spikes of the H1cell are precisely coupled only to rapid velocity changes. At less transient episodes of time-dependent motion stimuli, spikes are time-locked to motion with a smaller temporal precision. As a consequence of inevitable temporal lowpass filters involved in motion detection (e.g. Reichardt 1961; Borst and Egelhaaf 1989; Egelhaaf and Borst 1993b), the response fluctuations induced even by velocity fluctuations with broad frequency spectrum contain most power below $20 \mathrm{~Hz}$. (Haag and Borst 1997, 1998; Warzecha et al. 1998). In general, slow membrane potential fluctuations are less effective in evoking an action potential than fast rises of the membrane potential (Johnston and Wu 1995). Indeed, fluctuations of the membrane potential above approximately $30 \mathrm{~Hz}$ have been reported in various systems, including the fly, to be more effective in eliciting spikes time-locked to a stimulus than less transient fluctuations (Mainen and Sejnowski 1995; Haag and 
Borst 1996; Nowak et al. 1997). This suggests that only very fast velocity changes lead to transient depolarizations that are sufficient to elicit precisely timed action potentials. Otherwise, the precise timing of spikes seems to be primarily governed by stochastic fluctuations of the membrane potential and the neuronal response is coupled to the motion stimuli on a coarser timescale.

Where do these stochastic fluctuations originate? In principle, the stochastic component observed in the responses of tangential cells could arise anywhere in the visual system starting from photon noise and the stochastic nature of photon absorption down to the level of the tangential cells themselves. It has been proposed that the performance of fly motion sensitive neurones in the third visual neuropil is limited by the photoreceptor noise (Bialek et al. 1991; de Ruyter van Steveninck and Bialek 1995). On the other hand, there is evidence that, depending on the light level, the synapse between photoreceptors and the first-order visual interneurones may also contribute a considerable amount of noise to the signal (Laughlin et al. 1987; Juusola et al. 1996; de Ruyter van Steveninck and Laughlin 1996). Apart from these noise sources in the periphery of the visual system, subsequent processing steps may also be noisy, although their significance in this regard has not yet been analysed. In addition to all these noise sources, the reliability of encoding of motion stimuli may also be affected by noise originating in the tangential cells themselves.

Whether noise intrinsic to the motion-sensitive tangential neurones mainly determines the exact timing of action potentials can be estimated by recording simultaneously the activity of two tangential cells which receive their input to a large extent from common retinotopically organized input elements. If the temporal jitter in the occurrence of spikes was primarily caused by noise originating in the common motion pathway peripheral to the tangential cells rather than by noise intrinsic to the tangential cells themselves or their input synapses, most spikes of the two cells should coincide. In contrast, when noise sources intrinsic to the tangential cells are most decisive, the spike activity of two tangential cells should not be significantly correlated.

The H1- and the H2- cell, two spiking tangential cells in the lobula plate of the fly, are thought to share large parts of their motion-sensitive input elements and not to be synaptically coupled to each other. Both neurones have largely overlapping receptive fields and the same preferred direction of motion (Hausen 1981). The mean activity of the H2-cell is lower than that of the H1-cell. The crosscorrelation between simultaneously recorded responses of the two neurones to white-noise velocity fluctuations, reveals a narrow peak (Fig. 7a). Both neurones are able to generate spikes with a much higher temporal precision than is expected on the basis of their time-coupling to the motion stimulus. This becomes obvious when we compare the CCGs of the simultaneously recorded and the randomly shuffled spike trains of both cells (Fig. 7). This conclusion is further corroborated by recordings from the $\mathrm{H} 1$ - and H2-cell during stimulation with constant-velocity motion. The CCG of the simultaneously recorded activity reveals a similar peak as obtained for transient motion stimulation. Since the synchronicity 
of spikes is not elicited by the motion stimulus, we conclude that it has its origin in a common noise source in the peripheral motion pathway or in the stochastic nature of light (for details, see Warzecha et al. 1998). Obviously, the spike generating mechanism does not introduce much jitter in the timing of spikes. This conclusion is in accordance with previous results obtained in other systems (Calvin and Stevens 1968; Mainen and Sejnowski 1995).
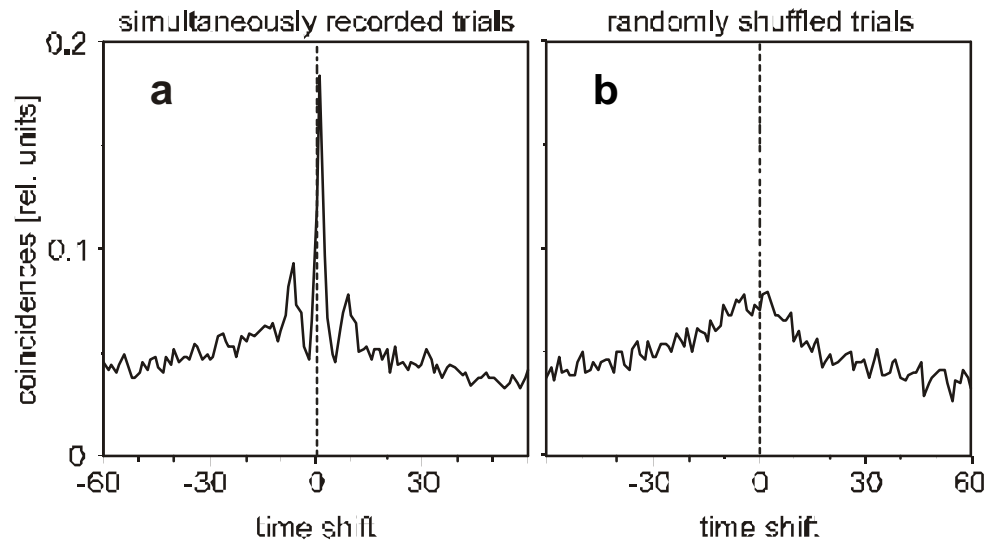

Fig. 7 Synchronization of spikes in neurones with common synaptic input. Crosscorrelograms between the simultaneously recorded responses of the $\mathrm{H} 1$ - and $\mathrm{H} 2$-neurone (a) and shuffled CCGs of the same number of pseudorandomly chosen $\mathrm{H} 1$ - and $\mathrm{H} 2$-responses that were not recorded simultaneously but obtained from repetitive stimulation with the same dynamic velocity stimulus (b). CCGs were normalized to the square root of the product of the peak values in the autocorrelograms of the $\mathrm{H} 1$ - and $\mathrm{H} 2$-cell. An ordinate value of 1 indicates that the responses of both cells are identical at a temporal resolution of $1.1 \mathrm{~ms}$. This value cannot be reached, even if all spikes of the $\mathrm{H} 2$-cell coincide with a spike of the $\mathrm{H} 1$-cell, because the $\mathrm{H} 2$-cell generates action potentials less frequently than the $\mathrm{H} 1$-cell. (a) The narrow peak illustrates that the $\mathrm{H} 1$ - and the H2-cell are able to time-lock spikes to input fluctuations with a millisecond precision. The peak in the CCG is slightly shifted indicating that the $\mathrm{H} 1$ spikes tend to precede spikes of the $\mathrm{H} 2$ neurone by $1.1 \mathrm{~ms}$. (b) The broad and flat peak in the randomly shuffled CCG shows that both neurones do not generate spikes in response to motion with a millisecond precision (for details, see Warzecha et al. 1998).

Taken together these results indicate that most spikes elicited by white-noise velocity fluctuations are not precisely time-coupled to stimulus-induced membrane potential fluctuations. Instead, spikes usually time-lock to fast fluctuations in the membrane potential that are not induced by the stimulus but are stochastic. Only, when the stimulus-induced response component is sufficiently transient may precise time-locking to the stimulus occur, as can be elicited by sudden velocity changes. These conclusions have been further corroborated by model simulations (Kretzberg et al., personal communication). Similar conclusions have been drawn for directionally selective neurones in area MT of monkeys. Here the visual 
stimulus also causes the neurone to modulate its spike rate on a coarse time scale consistently from trial to trial, whereas the actual timing of individual spikes has been concluded to be effectively random (for review, see Shadlen and Newsome 1998).

\section{Performance of spiking and graded potential neurones in the encoding of visual motion}

Fly tangential cells encode visual motion either by sequences of action potentials (e.g., H1-cell) or by graded changes in the membrane potential that may be superimposed by spike-like events (e.g., HS-cells; see also Hengstenberg 1977; Hausen 1982a; Haag et al. 1997; Haag and Borst 1998). These two response modes are not only characteristic of fly tangential neurones but are commonly employed at various processing stages of different sensory modalities in both vertebrates and invertebrates (for review, see Roberts and Bush 1981). Are there any differences between these coding strategies with respect to the reliability with which the respective neurones represent and process motion information?

The spiking and the graded response mode were compared on the basis of responses of the $\mathrm{H} 1$ - and HS-cell by using several different criteria which address the following questions:

- How reliably do the two types of neurones signal the presence of a motion stimulus?

- How fast can they signal a motion onset?

- How many different stimulus states can be discriminated on the basis of the activity of both types of neurones?

- How much information about stimulus velocity is represented by spiking and graded potential tangential cells?

How reliably is a motion stimulus detected? As a criterion for comparing the performance of both response modes, the reliability was determined with which one can detect a constant velocity stimulus on the basis of the responses of the H1and the HS-cell. The neuronal activity recorded before and during motion stimulation was evaluated by a statistical procedure derived from signal-detection theory (Green and Swets 1974). This procedure assumes a hypothetical ideal observer who looks through a pair of windows at the neuronal activity before and during motion stimulation. The ideal observer does not know which window belongs to which stimulus condition. Rather it is the observer's task to carry out this assignment on the basis of the integrated activity within each of the windows. The ideal observer bases his/her decision on the knowledge that, on average, the stimulus leads to an increased activity. If this procedure is applied to many responses elicited by identical stimulation, the proportion of correct decisions can be determined as a statistical measure of reliability. A value of 0.5 indicates that false and correct decisions occur equally often, i.e. the activity during motion stimulation cannot be 

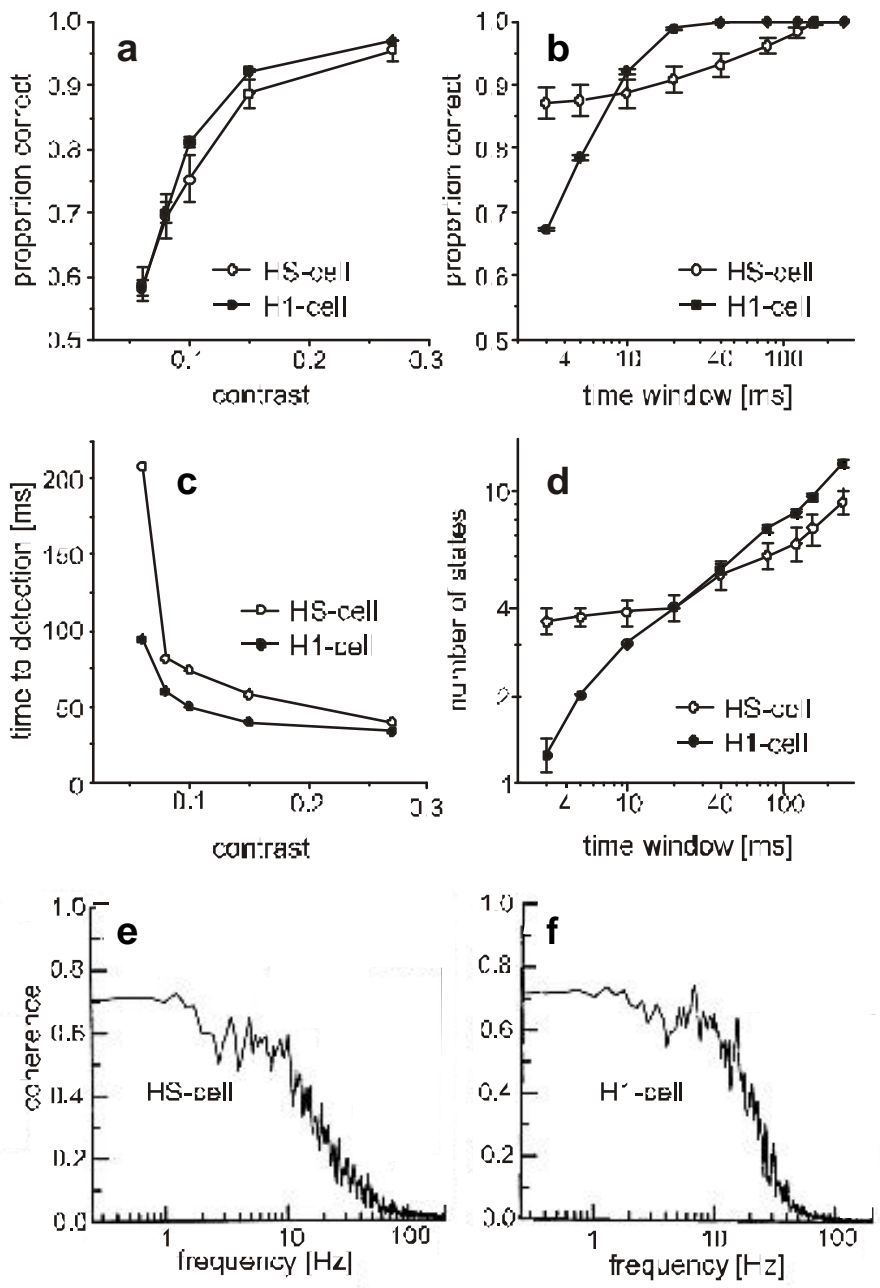

Fig. 8 Performance of HS-cell which mainly responds with graded changes in its membrane potential, and $\mathrm{H} 1$-cell that generates regular action potentials. a, b Proportion of correct decisions with which a motion stimulus can be detected on the basis of the steady state responses of the H1-cell (filled circles) and the HS-cell (open circles), plotted as a function of pattern contrast (a, time window $10 \mathrm{~ms}$ ) and the time window within which the neuronal activity was integrated (b, contrast 0.15 ). c Time to detection as a function of pattern contrast was determined within an $80 \mathrm{~ms}$ time window as the interval between the motion onset and the first time instant $75 \%$ of correct decisions was reached. The time to detection was not calculated for each cell separately because at low contrasts a reliability level of $75 \%$ was only reached for some cells. d Number of discriminable states as a function of the width of the time window (methods described in text). (a-d): The responses of 8 H1-cells and 9 HS-cells were evaluated. For each H1-cell (HS-cell) responses to 20 (5-10) presentations of the same stimulus were taken into account. Error bars denote SEMs. (Modified from Warzecha 1994). e, f Average coherence as a function of stimulus frequency, calculated from the responses of 6 HS-cells (e) and 10 H1-cells (f) with 5 to 20 
discriminated from the resting activity. A value of 1 is obtained if there are only correct decisions (for details of the method, see Warzecha and Egelhaaf 1998). For the comparison of the performances of the H1- and HS-cell, pattern contrast was varied to cover large parts of the neurones' activity range.

For both the H1- and the HS-cell the proportion of correct decisions increases in a similar way with increasing pattern contrast and thus an increasing mean response amplitude (Fig. 8a). The proportion of correct decisions also increases with increasing size of the time window within which the neuronal activity is integrated, because large time windows average out the stochastic component to a larger extent than do small time windows (Fig. 8b). For small time windows the responses of the HS-cell are more reliable than those of the H1-neurone. For intermediate window sizes the responses of the H1-cell lead to higher proportions of correct decisions. Large time windows (above $100 \mathrm{~ms}$ ) result in a very reliable detection of the motion stimulus for both cell types.

How fast is a motion onset detected? The time it takes to detect the onset of a motion stimulus is used as a further criterion to compare the performance of the two types of tangential cells. To evaluate how long it takes until the motion onset is reliably signalled the proportion of correct decisions is determined as a function of time. The "time-to detection" is defined as the time interval between the motion onset and the moment when a reliability of $75 \%$ correct decisions is reached (for details of the method, see Warzecha and Egelhaaf 1998). For both the H1- and the HS-cell the time-to-detection decreases considerably with increasing pattern contrast reaching reliable responses for high-contrast motion stimuli already after about $30 \mathrm{~ms}$ (Fig. 8c). The graded potential neurone does not signal the motion onset any faster than the spiking neurone. Within limits, this result does not much depend on the size of the time window with which the performance of the neurones was assessed (for details, see Warzecha 1994).

How many stimulus states can be discriminated? As another criterion to assess the performance of spiking and graded potential neurones, the number of stimulus states has been determined that can be discriminated with a reliability of $75 \%$ on the basis of the neuronal activity. Again the concept of an ideal observer assigning responses to one of two stimuli was used. The stimuli which had to be discriminated were constant velocity stimuli which differed in their contrast. For each contrast the distribution of the responses to repeated stimulation was determined by integrating for each individual response the steady-state neuronal activity

individual responses per cell. The coherence indicates how much information about the stimulus velocity is preserved in the neuronal responses. (Modified from Fig. 5, Haag and Borst 1997). Due to the low spontaneous activity of the H1-cell, the activity is modulated over a much larger range by motion in the preferred than by motion in the null direction, whereas the HS-cell is not restricted in this way because it hyperpolarizes during motion in null direction. To enable a direct comparison between the performance of a spiking and a graded response cell, the original velocity trajectory was replayed to the $\mathrm{H} 1$-cell also in a mirror-symmetrical version so that every displacement of the stimulus pattern occurred in the direction opposite to the original one. The spike trains of the H1-cell to both versions of the stimulus trace were combined into a single response with positive and negative spikes for calculation of the coherence function. 
within a given time window. This was also done for the resting activity. The distribution obtained for the resting activity is used as the first reference distribution. A response distribution is then sought such that a randomly drawn sample of this distribution can be discriminated with a reliability of $75 \%$ from a randomly drawn sample of the reference distribution. Since the response distributions could only be determined for a limited number of contrasts, samples of adjacent response distributions are unlikely to be discriminable with a reliability of exactly $75 \%$. Hence, intermediate distributions were interpolated from the experimentally determined distributions obtained with the next higher and next lower contrast. Once the distribution was determined that can be discriminated with a reliability of $75 \%$ from the resting distribution, this distribution was used as the next reference distribution. The whole procedure was repeated until the response distribution was reached that is associated with the highest contrast (for details, see Warzecha 1994). For small time windows more stimulus states can be discriminated on the basis of the HS-cell responses than on the basis of the responses of the H1-cell. For larger time windows the performance of both cells does not differ much, although the spiking neurone may be able to encode slightly more states than the graded potential cell (Fig. 8d). Hence, with respect to the number of stimulus states which can be discriminated on the basis of the neuronal activity, graded potentials have advantages over spikes only on a short time scale.

How much information about stimulus velocity is preserved in spiking and graded potential neurones? The performance of spiking and graded potential tangential neurones was further compared by determining how well the velocity of a randomly fluctuating motion stimulus is represented by the two neurones with different response modes (Haag and Borst 1997). The reverse reconstruction method (Eggermont et al. 1983; Bialek et al. 1991; Theunissen et al. 1996; Borst and Theunissen 1999) was used to reconstruct the stimulus velocity from the neuronal responses. The analysis was done in the frequency domain yielding the best linear filter that transforms the neuronal responses into the stimulus velocity. The coherence between the real and the reconstructed stimulus calculated as a function of frequency served as a measure to quantify how well either cell can represent the velocity. Up to oscillation frequencies of approximately $10 \mathrm{~Hz}$ the stimulus velocity is well preserved in the responses of both types of neurones as long as the pattern moves into the preferred direction of motion. However, the spike activity of the H1-cell does not very well encode the velocity of a pattern moving in the cell's null direction. Apart from this difference that results from the low spontaneous activity of the H1-cell and the accordingly limited dynamic range for motion in the null direction, both the HS-cell and the H1-cell perform almost indistinguishably (Figs. 8e and f). At higher oscillation frequencies and larger stimulus amplitudes the coherence between stimulus velocity and the time course of the neuronal responses becomes much smaller (Haag and Borst 1997). This deviation from representing stimulus velocity is only partly due to stochastic fluctuations in the neural responses. Since the stimulus-induced responses of fly tangential neurones do not only depend on the velocity but also on its temporal 
derivatives (Egelhaaf and Reichardt 1987; see also Section 4.1), it is to be expected that the coherence between stimulus velocity and the neuronal responses should decrease for more transient motion stimuli. Irrespective of these complications, it is important to note in the present context, that the spiking and the graded potential neurone perform in basically the same way in representing dynamical motion stimuli in the cells' preferred direction.

In conclusion, as judged by a wide range of criteria, a spiking and a graded potential neurone that are located at the same level of information processing in the fly's motion pathway resemble each other closely with respect to their reliability in representing motion information. Only at a fine timescale are there pronounced differences in the performances of both response modes, where the graded potential cell may be superior over the spiking one (Figs. 8a-d). Of course, the possibility that other graded and spiking neurones perform differently to the HS- and H1-cell, respectively, cannot be excluded. Moreover, by using other criteria to assess the performance of spiking and graded potential neurones more significant differences between both response modes may emerge. A final assessment with respect to the still open problem why some neurones convey information by graded potentials whereas others do so by generating trains of spikes is only possible, if it is known which parameters of neuronal activity carry behaviourally relevant information (e.g. Liebenthal et al. 1994).

\section{Ecological constraints for neuronal representation of motion}

The temporal precision required to represent visual motion mainly depends on the dynamical properties of the motion stimuli that an animal encounters when solving a particular task. An animal is confronted with visual motion in two kinds of situations, either, when it views moving objects or when it moves through its eviron ment (see Eckert and Zeil, this volume). In the latter case the images of objects in the surroundings move across the retina of the animal even if these objects are stationary. The dynamical properties of retinal motion do not only depend on the time-varying velocity of the moving animal or of objects moving in its visual field, but also on the three-dimensional layout of the environment. Changes in the retinal velocity are thus not necessarily due to velocity changes of the moving animal or of objects moving in its visual field. During translation of the animal, they may also have their cause in changing distances between the eye and stationary or moving objects in the surround. Changes in the direction and speed of an animal's self-motion are limited by inertia and friction. The relative contribution of these two factors are influenced by the size of the animal and the substrate on or in which it moves. As a consequence of these physical constraints, retinal motion stimuli will not change their direction at arbitrarily high frequencies under natural conditions. 

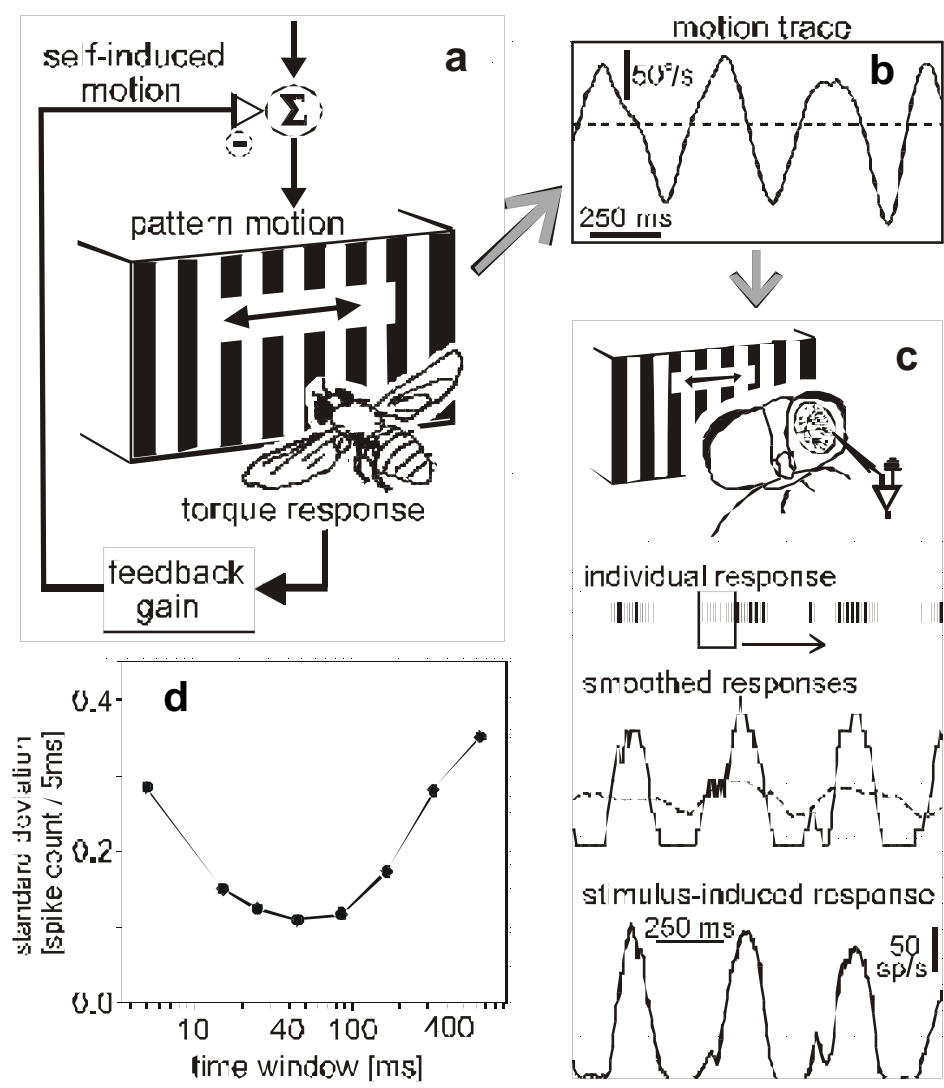

individual response

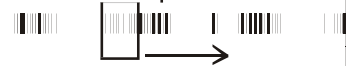

smouthed responses

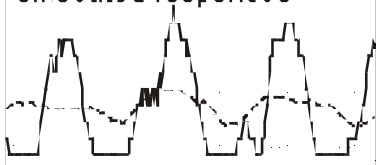

st mulus-induced response

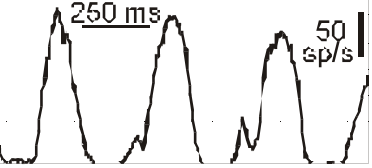

Fig. 9 Behaviourally generated motion stimuli and their representation by the H1-cell. a Generation of the motion traces in a behavioural closed-loop situation in a flight simulator. The fly is tethered to a torque compensator (not shown) which allows to determine the fly's instantaneous yaw torque. The visual consequences of self-motion are simulated by transforming the torque signals into image displacements on a CRT screen. b Short section of a motion trace generated by a fly in the situation illustrated in (a). $\mathbf{c}$ In the upper part a fly looking at a monitor screen is shown schematically from behind with a hole cut into its head capsule and an electrode inserted into the brain. Behaviourally generated motion traces were replayed to the fly while recording the spike activity of the H1-cell in the right half of the brain. A section of a spike train obtained in this way is shown in its original form (with each vertical line indicating the time of occurrence of a spike; upper trace) and in two temporally smoothed versions (middle traces), obtained by integrating the number of spikes within a time window that was sliding across the spike trains. The size of the time window was either $40 \mathrm{~ms}$ (middle trace, solid line) or $320 \mathrm{~ms}$ (middle trace, dashed line). The bottom trace shows the corresponding section of the stimulus-induced response component as obtained by averaging over 40 individual responses to the identical motion trace. $\mathbf{d}$ Similarity between smoothed individual and stimulus-induced responses given by the standard deviation: For each time window the squared difference between each smoothed individual response and the stimulus-induced response was averaged across time and across trials. For each cell the square root of this value was determined. The analysis was performed at a temporal resolution of $5 \mathrm{~ms}$. Values indicate means of three cells. The stimulus-induced response component elicited by behaviourally generated motion stimuli can be estimated best for each instance of time from noisy neuronal signals if these are smoothed by medium-sized time windows (40-100 ms). 
Not much is known about the dynamical properties of behaviourally relevant motion stimuli. They can only be characterized by analysing the behaviour of the animal and by reconstructing the optic flow encountered by the animal in the real world. In the fly, a first modest attempt has been made in the context of optomotor course control to analyse neural coding of visual motion stimuli that were generated by the fly's own actions and reactions. The analysis was done with a flight simulator operated under closed-loop conditions where the tethered flying fly could control by its yaw torque the horizontal displacements of its retinal input (Fig. 9a).

When the visual consequences of a disturbance of the fly's flight course are simulated by displacing the stimulus pattern with a constant velocity in one direction, flies are able to compensate for this disturbance to a large extent. Nonetheless, the torque and thus the retinal motion is characterized by pronounced temporal fluctuations (Fig. 9b, Warzecha and Egelhaaf 1996). The power spectrum of these velocity fluctuations is characterized by low frequencies of up to 5 Hz (Heisenberg and Wolf 1988; Warzecha and Egelhaaf 1997). The motion stimuli generated by a behaving fly were replayed in electrophysiological experiments while the activity of the spiking H1-cell was recorded (Fig. 9c). The spike activity was modulated depending on the direction and speed of pattern motion, with some jitter in the timing of spikes. As a consequence, the spike rate as obtained by averaging over many presentations of the same behaviourally generated motion trace varied smoothly, similar to the responses elicited by white-noise velocity fluctuations (for details, see Warzecha and Egelhaaf 1997).

How well do these neuronal responses encode the behaviourally generated motion stimuli? This question relates to two aspects which should not be confounded, i.e. (i) the parameters of the motion stimulus that are encoded by the neuronal responses and (ii) the reliability with which this is done. The first of these aspects can be analysed by reconstructing a certain feature of the motion stimulus from the time-dependent spike trains. This approach has been employed for dynamical motion stimuli, though not behaviourally generated ones, by determining the linear filter that leads, on the basis of the individual response traces, to an optimal estimation of the time-dependent velocity of motion (see Section 4.1). In this way, it is possible to assess how well individual responses represent a particular stimulus parameter, such as pattern velocity. However, this approach does not easily allow us to assess how reliably motion information is processed because deviations of the estimated velocity from the real pattern velocity can have two reasons. One reason is the nonlinear relationship between the velocity of the motion stimulus and the neuronal responses (see Section 4.1.). The other reason is neuronal noise (Warzecha and Egelhaaf 1997; Haag and Borst 1997). The reliability with which motion stimuli are encoded, i.e. the second of the above-mentioned aspects, has been assessed for the behaviourally generated motion stimuli obtained in the context of optomotor course control by relating the individual response traces to the stimulus-induced response component, i.e. to the instantaneous spike rate. If there were no jitter in the timing of spikes and the responses 
were precisely coupled to the motion stimulus, the individual responses and the stimulus-induced response component should look alike. Obviously, this is not the case (Fig. 9c) and it is not possible to predict the stimulus-induced response component very well for each instant of time from the instantaneous activity of individual responses. A much better prediction of the stimulus-induced response component is possible for each instant of time, when the individual spike trains are temporally smoothed to some extent (compare the different traces in Fig. 9c). It is obvious that, if the time window within which spikes are temporally averaged is too small, the filtered individual responses and the stimulus-induced response component differ greatly. On the other hand, if the time window is too large, fluctuations of the response component that are elicited by the motion stimulus are much attenuated. Hence, there should be an optimal time window leading to the best representation of the stimulus-induced response component on the basis of individual responses or, in other words to the best prediction of the spike rate for each instant of time. The similarity between the filtered individual responses and the stimulus-induced response component was assessed in two ways (i) on the basis of an information theoretic approach (Warzecha and Egelhaaf 1997, see Appendix) and (ii) by determining for each instant of time the square root of the mean squared differences between the filtered individual response traces and the stimulus-induced response component ("difference measure"). Both approaches led to the same result: As is shown in figure $9 \mathrm{~d}$ for the difference measure, the stimulus-induced response to motion generated by the fly during optomotor course stabilization can be estimated best for each time instant when individual spike trains are temporally smoothed within time windows of some tens of milliseconds, that is on a timescale on which the exact timing of individual spikes does not matter much. This finding may be not very surprising, as in this task the motion stimuli and, accordingly, the motion-induced response component did not contain much power for oscillation frequencies above 6-10 Hz. The optimal time window will be smaller when the motion stimuli and thus the stimulus-induced response component contain higher frequencies than the stimuli generated by the behaving fly in the context of optomotor course control and the corresponding responses used here for analysis (see Appendix).

Although the retinal image displacements are expected to be much smaller while the fly tries to stabilize its course against disturbancies than during voluntary turns, the significance of the findings summarized in figure 9 will be qualified in the following. First of all, it is necessary to exclude the possibility that the relatively slow dynamics of the behaviourally generated motion traces is the consequence of the lowpass properties of the flight simulator (Warzecha and Egelhaaf 1997). Since in another behavioural context (object detection and fixation), that was also analysed using the flight simulator, much faster responses are generated by the fly than during optomotor course control (Egelhaaf 1987; Zanker et al. 1991; Kimmerle et al. 1997), the dynamics of the flight simulator are not the main determinant of the dynamics of the behaviourally generated optomotor responses. In addition, increasing or decreasing the cut-off frequency of the lowpass filter in 
the flight simulator by a factor of two does not change the dynamics of the optomotor responses in any obvious manner (Warzecha and Egelhaaf 1997). Moreover, there is evidence that the signals of the output elements of the third visual neuropile are temporally lowpass filtered somewhere between the third visual neuropile and the steering muscles mediating optomotor course stabilization (Egelhaaf 1987, 1989). The time constant estimated for this lowpass filter is much larger than that of the flight simulator. Hence, the relatively slow dynamics of image motion during optomotor course stabilization, as analysed in the flight simulator, can be concluded to be a genuine property of this control system. Of course, it is not easily possible to derive from the experiments done with the flight simulator the functional significance of the optomotor responses under free-flight conditions. This problem cannot be resolved at present, since no one has analysed under free-flight conditions optomotor course stabilization in blowflies (Calliphoridae) and houseflies (Muscidae) on which most behavioural experiments in the flight simulator were done. However, there is evidence that in several insect species optomotor course stabilization is comparatively slow also under free-flight conditions (Collett 1980; Farina et al. 1995; Kern and Varjú 1998). Although hoverflies, for instance, can execute tremendously virtuosic flight manoeuvres and can change their flight direction very rapidly (Collett and Land 1975), their compensatory turning responses - even under free-flight conditions - have a temporal lowpass characteristic which leads to attenuation of the optomotor response at frequencies above $1 \mathrm{~Hz}$ (Collett 1980) and thus in a similar range as found in the flight simulator with houseflies (Egelhaaf 1987). Hence, there might exist common computational reasons for optomotor course stabilization to operate on a relatively slow timescale (for discussion see Collett 1980; Egelhaaf 1987).

The temporal characteristics of the retinal image displacements may differ considerably for behaviour other than optomotor course stabilization. The relevant timescales of retinal image motion are thus likely to vary over a wide range in different behavioural contexts. Unfortunately, there are only few examples where information about the dynamics of behaviourally relevant motion stimuli is available. One such example is optomotor position stabilization of the hummingbird hawkmoth which hovers almost stationarily in front of flowers while sucking nectar. Flowers, on which the hawkmoth is feeding, were found to wiggle in the wind at frequencies between about 1 and $2 \mathrm{~Hz}$ and thus change their direction of motion on a relatively slow timescale (Farina et al. 1994). Another example are the retinal image displacements experienced by solitary wasps while acquiring a visual representation of the environment of their nest in systematic orienting flights. During these flights the animals fly in ever increasing arcs around their nest, thereby changing their direction of motion and, thus, the overall direction of retinal image displacements at frequencies in the range of $0.5 \mathrm{~Hz}$. During these movements the wasps do not change the orientation of their body axis smoothly but rapidly in a saccade-like manner. Nevertheless the prevailing frequencies in the angular velocity profiles hardly lie above $10 \mathrm{~Hz}$ (Zeil 1993; Voss and Zeil 1998). Similar saccade-like turns can be observed during free-flight manoeuvres 
of flies (Wagner 1986a,b; Land 1993). In recent experiments where the turning dynamics of freely flying flies could be analysed at very high spatial and, in particular, temporal resolution, even the most rapid turns appear to take at least $15 \mathrm{~ms}$ (van Hateren and Schilstra 1999; Schilstra and van Hateren 1999). So far, no one has recorded neuronal responses to these rapid velocity changes. However, as judged by the responses to more conventional laboratory stimuli, it is well conceivable that the initial spikes elicited by changing the direction of the body axis as rapidly may well be timed precisely on a millisecond timescale (see also de Ruyter van Steveninck et al., this volume).

In this context it needs to be reiterated that not all visual motion stimuli an animal is confronted with in real life are as transient. The rapid turns as described above are certainly just one extreme. Also chasing behaviour of male flies in the context of mating behaviour is extreme with respect to speed and virtuosity. Since in many free-flight studies - for technical reasons - the flies were restricted to fly within a relatively small space, requiring frequent changes of flight direction, we do not know anything about the dynamics of retinal motion in other behavioural contexts, for instance, during cruising flight over distances of some tens to hundreds of meters or when the animal compensates for a disturbance of its flight course. Hence, no firm conclusions concerning the relevant timescales of encoding of motion information in normal behavioural situations and in a variety of relevant behavioural contexts of a fly are possible at present.

\section{Conclusions}

All information about the outside world available to an animal is somehow encoded in the temporal activity patterns of its neurones. A variety of approaches has been employed to demonstrate that for directionally selective, motion sensitive neurones in the fly different aspects of visual motion stimuli can be derived even from individual responses. (i) The onset and presence of motion can be detected reliably (Section 6). Under certain conditions, even the pattern velocity can be reconstructed from individual spike trains (see Section 4.1; Bialek et al. 1991; Haag and Borst 1997). (ii) It is possible to discriminate between spatial displacements which differ by less than the spacing of two photoreceptors on the basis of the timing of the first spike or the first pair of spikes generated after stimulus presentation (de Ruyter van Steveninck and Bialek 1995). (iii) Pairs of spikes may carry considerably more information about the motion stimulus than the sum of the information contributed by each spike separately (de Ruyter van Steveninck and Bialek 1988).

Although these findings clearly reveal that temporal patterns of neural activity provide significant information about visual motion, they do not allow us, without further qualification, to make inferences about the timescale on which spikes are locked to the timecourse of motion. We have argued that the real-time 
performance of motion-sensitive neurones in the fly visual system is constrained by various properties of the underlying neuronal machinery. As a consequence of the biophysical properties of neurones, action potentials time-lock to fast membrane potential changes with a higher temporal precision than to slower changes. Hence, spikes are likely to time-lock on a millisecond scale to rapid stochastic membrane potential changes which dominate in the postsynaptic potentials of the cells, if the stimulus-induced membrane potential changes are not sufficiently fast (see Sections 4.3 and 5). Although fast velocity transients are much attenuated by the motion pathway as a consequence of temporal filters inherent to the mechanism of motion computation, it is possible to generate rapid velocity changes that lead to a precise time-locking of spikes on a timescale of 1-2 ms. Whether changes in the optic flow occurring in natural flight situations are transient enough to lead to precise time-locking of spikes needs to be analysed in future experiments. As mentioned above, there are indications that this might well be the case under special conditions (de Ruyter van Steveninck et al., this volume). Nonetheless, most spikes elicited by broad-band velocity fluctuations are coupled to the stimulus on a timescale of some tens of milliseconds. We thus conclude that the timing of spikes on a millisecond scale is governed to a large extent by stochastic membrane potential fluctuations that are more transient than the membrane potential fluctuations elicited by most episodes of visual motion traces. As a consequence, the stimulus-induced neuronal response component elicited by motion stimuli generated by the behaving fly while stabilizing its course against disturbances can be estimated best for each instant of time on the basis of individual response traces, if these are temporally smoothed on a timescale of several tens of milliseconds (see Section 7).

At first sight these findings appear to contradict recent results of an elaborate information theoretic analysis of responses of the H1-neurone by Strong et al. (1998). Their study has shown that spike trains of the H1-cell elicited by broadband velocity fluctuations transmit increasingly more information about the stimulus when the spike responses are evaluated at an increasingly finer temporal resolution down to millisecond precision. It is puzzling why seemingly contradictory results have been obtained on the basis of the activity of the same neurone with, at least partly, the same type of stimuli, and both studies using a similar measure for the neurone's reliability. A close look, however, reveals that the results of both studies do not necessarily contradict each other. Instead, different aspects of encoding of motion information have been analysed. Strong et al. (1998) were looking at a given signal, i.e. the spike trains of the H1-cell, with different temporal resolutions and demonstrated that the information transmitted by the individual responses about the stimulus increases with the temporal resolution. In contrast, we compared with a given temporal resolution different signals, i.e. the individual spike trains and their temporally smoothed versions and demonstrated that the stimulus-induced response component can be estimated best from noisy individual spike trains if these are smoothed to some extent (Figs. 9c and d, see Appendix). 
The conclusions regarding the temporal precision with which spikes are locked to the stimulus raise a principal conceptual question. All inferences an animal can make about its outside world are necessarily based on the electrical activity evoked by its sensory input. Even if part of the incoming action potentials are time-locked very precisely to the stimulus, in the absence of additional information the animal has no chance to infer which of the action potentials can be relied on with respect to the timing of the stimulus and which are less reliable in this regard. It needs to be analysed whether there are means in the fly's motion pathway to make use of the information which is potentially carried by precisely timed spikes.

Although not much is known about the relevant timescales of visual motion an animal such as the fly encounters in normal life, it appears to be plausible to assume that the dynamics of retinal motion covers a wide range and may vary systematically and quite a lot with the behavioural context (see Section 7). Hence, it is hard to define the computational needs for a temporal coupling of spikes to visual motion stimuli on a millisecond scale. However, very precise timing of spikes to the sensory input is necessary, in other computational contexts, for instance when an object is to be localized on the basis of acoustic or electrical signals or when the activity of flight motor neurones is coupled to the temporal phase of the wingbeat as is the case in flies. Accordingly, very rapid stimulusinduced membrane potential fluctuations and, thus, a very precise time-locking of spikes to stimuli are found in neurones of the fish electrosensory system involved in object detection (Kawasaki 1993), in the system mediating acoustic sound localization of vertebrates (Carr 1993) and the mechanosensory system of flies involved in coupling the activity of flight motor neurones to the temporal phase of wing beat (Fayyazuddin and Dickinson 1996). Motion vision systems that do not need to represent such high-frequency fluctuations under most behaviourally relevant stimulus conditions appear to be adapted to the more slowly changing stimulus-induced fluctuations they encounter in the real world. In fact, it has been suggested on the basis of the temporal tuning of motion-sensitive neurones in a variety of insect species, that the temporal filtering properties of motion detection systems are adapted to the lifestyle of the respective animal (O'Carroll et al. 1996). However, the quantitative characterization of naturally occuring motion stimuli poses many technical problems and has not been done in any detail so far. We are just beginning to quantify behaviourally relevant visual motion signals by reconstructing the time-dependent retinal input from video films taken of freely moving flies in a variety of behavioural contexts (Kern et al. 1999, 2000). It will be one of the challenges in the near future to investigate how and on what timescale motion stimuli that are encountered by the animal in natural situations are represented by its nervous system.

In conclusion, the relevant timescale needed for the encoding of motion information cannot be derived directly from information theoretic or system analytical approaches to responses elicited by artificial stimuli. Rather, it is essential also to take an ecological perspective to find out what stimuli an animal encoun- 
ters in real life. Only then it is possible to tell what might be really relevant for an animal and thus to understand what neural codes are used by a particular nervous system in a given task.

\section{Acknowledgements}

We thank our co-workers R. Kern, B. Kimmerle, H. Krapp and J. Kretzberg, for reading and discussing the manuscript. B. Kimmerle performed the experiment shown in figure $5 \mathrm{~b}$ which is gratefully acknowledged. J. Zanker and J. Zeil as well as two anonymous referees made many critical annotations to a previous version of the paper and, thus, helped to improve it considerably.

\section{Appendix}

\section{On the estimation of the stimulus-induced response component from individual spike trains: Misconceptions and misunderstandings}

Individual spike trains elicited by dynamical motion stimuli can be used to estimate the corresponding velocity trajectory or the stimulus-induced response component which is reflected in the time course of the mean spike rate (see Sections 4.1 and 7). The best estimates are obtained if the noisy individual spike trains are smoothed to some extent by appropriate temporal filtering (Bialek et al. 1991; Haag and Borst 1997; Warzecha and Egelhaaf 1997; see also Fig. 9c). Temporal filtering increases the similarity between the individual spike trains and the signal to be estimated only if the signals are not statistically independent at subsequent instants of time, but contain temporal correlations. If there are temporal correlations on a coarse timescale the time constant of the best filter is larger than when there are correlations mainly on a finer timescale. Accordingly, the time-constant of the filter can be expected to be smaller for rapidly varying stimuli than for slowly varying ones (see below). Although all this is already obvious from comparing the filtered spike trains with either the stimulus velocity (e.g., Fig. 2 in Bialek et al. 1991) or the stimulus-induced response component (see Fig. 9c), the quality of the estimation needs to be quantified, especially if the performance of different filters is to be compared. There are various ways how this comparison can be done. Perhaps the most straightforward way is to determine $\left(?^{2}=?[\mathrm{~s}(\mathrm{t})\right.$ $\left.\mathrm{s}_{\text {est }}(\mathrm{t})\right]^{2} \mathrm{dt}$, with $\mathrm{s}(\mathrm{t})$ representing either the time-dependent stimulus velocity or the stimulus-induced response component and $\mathrm{s}_{\mathrm{est}}(\mathrm{t})$ corresponding to the estimated signal based on temporally filtering the individual responses (see difference measure in Section 7 and Bialek et al. 1991).

In a previous account we employed another measure of how well the stimulus-induced response component can be estimated for each instant of time on the 
basis of the original and filtered individual responses (Warzecha and Egelhaaf 1997). To obtain a measure for the similarity we first determined $\mathrm{p}\left(\mathrm{s}_{\mathrm{i}} \mid \mathrm{r}_{\mathrm{j}}\right)$, the conditional probability with which a particular level of the stimulus-induced response component $\left(\mathrm{s}_{\mathrm{i}}\right)$ occurs given a particular individual response $\left(\mathrm{r}_{\mathrm{j}}\right)$, and $\mathrm{p}\left(\mathrm{s}_{\mathrm{i}}\right)$, the probability of occurrence of $\left(\mathrm{s}_{\mathrm{i}}\right)$. We then calculated the ratio of both terms $\mathrm{p}\left(\mathrm{s}_{\mathrm{ii}} \mathrm{r}_{\mathrm{j}}\right) / \mathrm{p}\left(\mathrm{s}_{\mathrm{i}}\right)$. This ratio will be unity and at its minimum, if the timedependent individual responses and the stimulus-induced response are completely unrelated, i.e. knowledge of $r_{j}$ does not help to predict $s_{i}$, (i.e. $p\left(s_{i l} r_{j}\right)=p\left(s_{i}\right)$ ). The ratio will be larger than 1 , if $r$ restricts the possible range of stimulus-induced responses (i.e. $\left.\mathrm{p}\left(\mathrm{s}_{\mathrm{i}}{ }^{\prime} \mathrm{r}_{\mathrm{j}}\right)>\mathrm{p}\left(\mathrm{s}_{\mathrm{i}}\right)\right)$. The more similar individual responses, $\mathrm{r}_{\mathrm{j}}$, are to the corresponding stimulus-induced response component, si, the better we can predict what $\mathrm{si}$ is likely to be when we know $\mathrm{r}_{\mathrm{j}}$ and the larger the ratio $\mathrm{p}\left(\mathrm{s}_{\mathrm{i}} \mathrm{r}_{\mathrm{j}}\right) / \mathrm{p}\left(\mathrm{s}_{\mathrm{i}}\right)$ will be. This ratio can be determined for each activity level of the stimulus-induced response component and each activity level of the individual responses and it can be used as a measure of the similarity between both signals. Using the logarithm of these ratios and taking into account that not all combinations of activity levels si and rj are equally likely to occur by weighting the different ratios by the probability of their occurrence, a measure of similarity is obtained which is formally equivalent to the so-called transinformation

$$
\mathrm{T}=?_{\mathrm{i}, \mathrm{j}} \mathrm{p}\left(\mathrm{s}_{\mathrm{i}}, \mathrm{r}_{\mathrm{j}}\right) \log _{2}\left[\mathrm{p}\left(\mathrm{s}_{\mathrm{i}} \mid \mathrm{r}_{\mathrm{j}}\right) / \mathrm{p}\left(\mathrm{s}_{\mathrm{i}}\right)\right] \text {. }
$$

The transinformation is the information which is transmitted by a signal, $r$, about another signal,s (e.g. Shannon and Weaver 1949; Rieke et al. 1997). In our previous study we plotted this transinformation in relative units (Fig. 3 in Warzecha and Egelhaaf 1997) because we only wanted to use the transinformation as a measure of the overall similarity between the stimulus-induced response component and the filtered or unfiltered individual responses. It should be noted that the transinformation was always determined with the same temporal resolution (time bins of $5 \mathrm{~ms}$ ) irrespective of how the individual spike trains were temporally smoothed. In accordance with the analysis shown in the present account (see Fig. 9), we obtained for the behaviourally generated motion traces the largest similarity, if the individual spike trains were smoothed by time windows of a width of 40 to $100 \mathrm{~ms}$ (Warzecha and Egelhaaf 1997). If the stimulus-induced response component contains higher frequency components than the one evoked by the behaviourally generated stimuli, the optimal time window is shifted to smaller values. This shift is illustrated in figure 10 for a model simulation. Two different motion-induced response traces were simulated by lowpass-filtering the same white noise sequence with a cut-off at either $80 \mathrm{~Hz}$ ("high-frequency response") or $20 \mathrm{~Hz}$ ("low frequency response"). These simulated time-dependent motion-induced responses were used to generate individual response traces (for details, see legend of Fig. 10). After smoothing individual spike trains to a variable extent, the transinformation was calculated separately for the high-frequency response and the corresponding individual spike trains on the one hand and the low frequency response and the corresponding spike trains on the other hand. For 
both the low- and the high-frequency responses the transinformation increases when individual responses are temporally smoothed before calculating the transinformation. The size of the optimal time window increases when the power of the motion-induced response is decreased in the high frequency range (compare triangles to circles in Fig. 10). This conclusion does not depend on the specific statistics of spike activity used for the simulations. Similar results are also obtained for experimental data. When instead of the motion stimuli generated by the behaving fly in the context of optomotor course control, white-noise stimuli as shown in figure 1 are presented to an $\mathrm{H} 1$-cell, the optimal time window for smoothing individual responses reduces from 80 to about $10 \mathrm{~ms}$ (Warzecha unpublished).

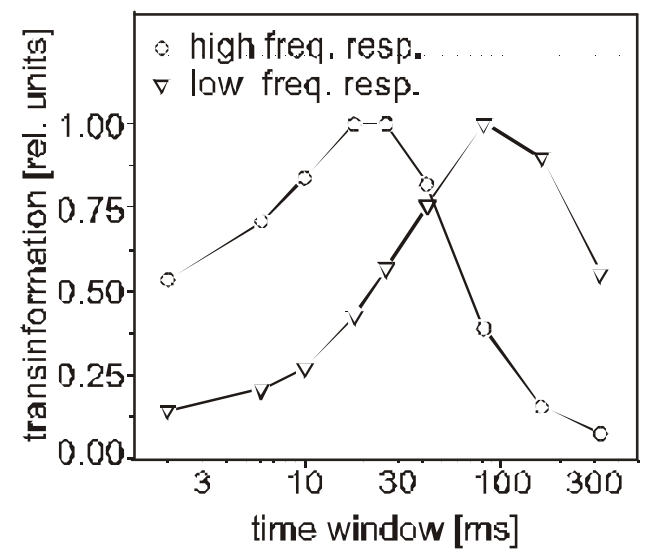

Fig. 10 Transinformation between artificially generated individual spike trains and the corresponding stimulus-induced response obtained by averaging 500 individual spike trains. In the first step of the simulation two different motion-induced responses and the corresponding individual spike trains were calculated. The responses differed with respect to their frequency content. A sequence of 5000 Gaussian distributed random numbers was generated corresponding to a response trace of $10 \mathrm{~s}$ with a temporal resolution of $2 \mathrm{~ms}$. To obtain the stimulus-induced response, the sequence of random numbers was temporally filtered by a first-order lowpass with a cutoff at either $80 \mathrm{~Hz}$ ("high frequency response") or $20 \mathrm{~Hz}$ ("low frequency response"). The high-frequency response was normalized to a range of values between 0 and 0.7 . The lowfrequency response was normalized by the same factor. These time-dependent responses determined the probability of spike generation as a function of time. The individual response traces were generated by comparing for each elementary time bin of $2 \mathrm{~ms}$ a uniformly distributed random number between 0 and 1 with the spike probability given by the respective stimulusinduced response in the respective time bin. If the spike probability exceeded the random number a spike was assigned to the corresponding time bin. 500 individual spike trains were generated in this way for each stimulus-induced response $(80 \mathrm{~Hz}$ and $20 \mathrm{~Hz})$. Prior to the calculation of the transinformation, the individual response traces were temporally smoothed by integrating the number of spikes within time windows of varying size (abscissa). For the calculation of the transinformation responses were subdivided into 11 equally sized activity classes (for details of the calculation, see Warzecha and Egelhaaf 1997). The transinformation was determined by taking into account the mean activity within $6 \mathrm{~ms}$ time bins. The transinformation was normalized to its maximum separately for the high frequency response (circles) and the low frequency response (triangles), respectively. The transinformation first increases with increasing temporal smoothing of the individual response traces, reaches an optimum and then decreases again. 
Having in mind the basic information theoretic theorem, that the information transmitted by a signal cannot be increased by temporally filtering the signal (Shannon and Weaver 1949), our result may, at first sight, appear paradoxical (see de Ruyter van Steveninck et al., this volume). There is no doubt that the information transmitted by a time-dependent signal cannot increase by filtering it. It should be noted, that one cannot determine the information content of timedependent signals in the way described above, unless it is ensured that the neuronal signals in subsequent time units that form the basis for calculating the information are statistically independent. This is a critical point which is usually not tested when calculating the information content of a neuronal signal. It is quite clear that the statistical independence is not given for subsequent time bins of $5 \mathrm{~ms}$ which formed the basis of our calculations. Moreover, it is also evident that temporal filtering the individual spike trains introduces correlations at a larger timescale and therefore fewer statistically independent activity levels per time unit. It should be noted that these correlations are not just a consequence of the filtering procedure but reflect correlations which are intrinsic in the time-modulated stimulus-induced response component. As a consequence of these correlations the stimulus-induced response component can be predicted more reliably on the basis of the individual responses, when these are filtered appropriately. However as a consequence of these inevitable correlations, it would be a severe misconception, to interpret the measure of similarity we used in our previous account (Warzecha and Egelhaaf 1997) in terms of information rates. One reason for this misinterpretation may be that the measure we used for assessing the similarity is called "transinformation" and thus suggests that the outcome of the calculations may be interpreted as information rate. Certain formulations in our previous account (Warzecha and Egelhaaf 1997) were not sufficiently precise in this regard and, thus, might have facilitated misinterpretations of our results.

\section{References}

Allen C, Stevens CF (1994) An evaluation of causes for unreliability of synaptic transmission. Proc Natl Acad Sci USA 91: 10380-10383

Bair W, Koch C (1996) Temporal precision of spike trains in extrastriate cortex of the behaving macaque monkey. Neural Comput 8: 1185-1202

Berry MJ, Warland DK, Meister M (1997) The structure and precision of retinal spike trains. Proc Natl Acad Sci USA 94: 5411-5416

Bialek W, Rieke F (1992) Reliability and information transmission in spiking neurons. Trends Neurosci 15: 428-433

Bialek W, Rieke F, de Ruyter van Steveninck R, Warland D (1991) Reading a neural code. Science 252: 1854-1857

Borst A, Egelhaaf M (1987) Temporal modulation of luminance adapts time constant of fly movement detectors. Biol Cybern 56: 209-215

Borst A, Egelhaaf M (1989) Principles of visual motion detection. Trends Neurosci 12: 297-306

Borst A, Theunissen FE (1999) Information theory an neural coding. Nature Neurosci 2:947-957

Britten KH, Shadlen MN, Newsome WT, Movshon JA (1993) Responses of neurons in macaque MT to stochastic motion signals. Vis Neurosci 10: 1157-1169 
Buchner, E (1984) Behavioural analysis of spatial vision in insects. In: Ali MA (ed) Photoreception and vision in invertebrates. Plenum Press, New York, London, pp 561-621

Buracas GT, Zador AM, DeWeese MR, Albright TD. (1998) Efficient dicrimination of temporal patterns by motion-sensitive neurons in primate visual cortex. Neuron 20: 959-969

Calvin WH, Stevens CF (1968) Synaptic noise and other sources of randomness in motoneuron interspike intervals. J Neurophysiol 31: 574-587

Carr CE (1993) Processing of temporal information in the brain. Ann Rev Neurosci 16: 223-243.

Collett TS (1980) Angular tracking and the optomotor response. An analysis of visual reflex interaction in a hoverfly. J Comp Physiol 140: 145-158.

Collett TS, Land MF (1975) Visual control of flight behaviour in the hoverfly Syritta pipiens L. J Comp Physiol 99: 1-66.

Egelhaaf M (1987) Dynamic properties of two control systems underlying visually guided turning in house-flies. J Comp Physiol A 161: 777-783

Egelhaaf M (1989) Visual afferences to flight steering muscles controlling optomotor response of the fly. J Comp Physiol A 165: 719-730

Egelhaaf M, Borst A (1989) Transient and steady-state response properties of movement detectors. J Opt Soc Am A 6: 116-127

Egelhaaf M, Borst A (1993a) A look into the cockpit of the fly: Visual orientation, algorithms, and identified neurons. J Neurosci 13: 4563-4574

Egelhaaf M, Borst A (1993b) Movement detection in arthropods. In: Wallman J, Miles FA (eds) Visual motion and its role in the stabilization of gaze, Elsevier, Amsterdam, London, New York, pp 53-77

Egelhaaf M, Reichardt W (1987) Dynamic response properties of movement detectors: Theoretical analysis and electrophysiological investigation in the visual system of the fly. Biol Cybern 56 69-87

Egelhaaf M, Warzecha A-K (1999) Encoding of motion in real time by the fly visual system. Curr Opinion Neurobiol 9: 454-460

Egelhaaf M, Hausen K, Reichardt W, Wehrhahn C (1988) Visual course control in flies relies on neuronal computation of object and background motion. Trends Neurosci 11: 351-358

Eggermont JJ, Johannesma PIM, Aertsen AMHJ (1983) Reverse-correlation methods in auditory research. Quart Rev Biophys 16: 341-414

Farina WM, Kramer D, Varjú D (1995) The response of the hovering hawk moth Macroglossum stellatarum to translatory pattern motion. J Comp Physiol A 176: 551-562

Farina WM, Varjú D, Zhou Y (1994) The regulation of distance to dummy flowers during hovering flight in the hawk moth Macroglossum stellatarum. J Comp Physiol 174: 239-247

Fayyazuddin A, Dickinson MH (1996) Haltere afferents provide direct, electrotonic input to a steering motor neuron in the blowfly, Calliphora. J Neurosci 16: 5225-5232

Geisler WS, Albrecht DG (1997) Visual cortex neurons in monkeys and cats: detection, discrimination, and identification. Vis Neurosci 14: 897-919

Gershon ED, Wiener MC, Latham PE, Richmond BJ (1998) Coding strategies in monkey V1 and inferior temporal cortices. J Neurophysiol 79: 1135-1144

Gestri G, Mastebroek HAK, Zaagman WH (1980) Stochastic constancy, variability and adaptation of spike generation: Performance of a giant neuron in the visual system of the fly. Biol Cybern 38: 31-40

Götz KG (1968) Flight control in Drosophila by visual perception of motion. Kybernetik 4: 199-208

Götz KG (1975) The optomotor equilibrium of the Drosophila navigation system. J Comp Physiol 99: 187-210

Götz KG (1991) Bewertung und Auswertung visueller Zielobjekte bei der Fliege Drosophila. Zool Jb Physiol 95: 279-286

Green DM, Swets JA (1974) Signal detection theory and psychophysics. Robert Krieger Publ Comp, Huntington, New York

Gur M, Beylin A, Snodderly DM (1998) Response variability of neurons in primary visual cortex (V1) of alert monkeys. J Neurosci 17: 2914-2920

Haag J, Borst A (1996) Amplification of high frequency synaptic inputs by active dendritic membrane processes. Nature 379: 639-641 
Haag J, Borst A (1997) Encoding of visual motion information and reliability in spiking and graded potential neurons. J Neurosci 17: 4809-4819

Haag J, Borst A (1998) Active membrane properties and signal encoding in graded potential neurons. J Neurosci 18: 7972-7986

Haag J, Theunissen F, Borst A (1997) The intrinsic electrophysiological characteristics of fly lobula plate tangential cells: II. Active membrane properties. J Comput Neurosci 4: 349-369

Harris RA, O'Carroll DC, Laughlin SB (1999) Adaptation and the temporal delay filter of fly motion detectors. Vision Res 39: 2603-2613

van Hateren JH, Schilstra C (1999) Blowfly flight and optic flow. II. Head movements during flight. J Exp Biol 202: 1491-1500

Hausen K (1981) Monocular and binocular computation of motion in the lobula plate of the fly. Verh Dtsch Zool Ges 74: 49-70

Hausen K (1982a) Motion sensitive interneurons in the optomotor system of the fly. I. The Horizontal Cells: Structure and signals. Biol Cybern 45: 143-156

Hausen K (1982b) Motion sensitive interneurons in the optomotor system of the fly. II. The Horizontal Cells: Receptive field organization and response characteristics. Biol Cybern 46: 67-79

Hausen K, Egelhaaf M (1989) Neural mechanisms of visual course control in insects. In: Stavenga D, Hardie R (eds) Facets of vision. Springer, Berlin, Heidelberg, New York, pp 391-424

Heisenberg M, Wolf R (1984) Vision in Drosophila. Springer, Berlin, Heidelberg, New York

Heisenberg M, Wolf R (1988) Reafferent control of optomotor yaw torque in Drosophila melanogaster. J Comp Physiol A 163: 373-388

Hengstenberg R (1977) Spike responses of 'non-spiking' visual interneurone. Nature 270: 338-340

Hengstenberg R (1982) Common visual response properties of giant vertical cells in the lobula plate of the blowfly Calliphora. J Comp Physiol 149: 179-193

Horstmann W, Egelhaaf M, Warzecha A-K (2000) Synaptic interactions increase optic flow specificity. Europ J Neurosci: in press

Ibbotson MR, Mark RF, Maddess T (1994) Spatiotemporal response properties of directionselective neurons in the nucleus of the optic tract and dorsal terminal nucleus of the wallaby, Macropus eugenii. J Neurophysiol 72: 2927-2943

Järvilehto M, Weckström M, Kouvalainen E (1989) Signal coding and sensory processing in the peripheral retina of the compound eye. In: Singh RN, Strausfeld NJ (eds) Neurobiology of sensory systems. Plenum Press, New York, London, pp 53-70

Johnston D, Wu M-S (1995) Foundations of cellular neurophysiology. MIT Press, Cambridge, MA

Juusola M, French AS, Uusitalo RO, Weckström M (1996) Information processing by gradedpotential transmission through tonically active synapses. Trends Neurosci 19: 292-297

Kawasaki M (1993) Temporal hyperacuity in the gymnotiform electric fish, Eigenmannia. Amer Zool 33: 86-93

Kern R, Varjú D (1998) Visual position stabilization in the hummingbird hawk moth, Macroglossum stellatarum L. I. Behavioural analysis. J Comp Physiol A 182: 225-237

Kern R, Lorenz S, Lutterklas M, Egelhaaf M (1999) How do fly interneurons respond to optic flow experienced in 3D-environments? In: Elsner N, Eysel U (eds) Proceedings of the 27th Göttingen Neurobiol Conf 1999. Thieme, Stuttgart, p 438

Kern R, Lutterklas M, Egelhaaf M (2000) Neural representation of optic flow experienced by walking flies with largely asymmetric visual input. J Comp Physiol A 186: 467-479

Kimmerle B, Srinivasan MV, Egelhaaf M (1996) Object detection by relative motion in freely flying flies. Naturwiss. 83: 380-381

Kimmerle B, Warzecha A-K, Egelhaaf M (1997) Object detection in the fly during simulated translatory flight. J Comp Physiol A 181: 247-255

Koenderink JJ (1986) Optic Flow. Vision Res 26:161-180

Krapp H (1999) Neuronal matched filters for optic flow processing in flying insects. In: Lappe M (ed) Neuronal processing of optic flow. Academic Press, San Diego, San Francisco, New York, pp 93-120

Land MF (1993) Chasing and pursuit in the dolichopodid fly Poecilobothrus nobilitatus. J Comp Physiol A 173: 605-613

Land MF, Collett TS (1974) Chasing behaviour of houseflies (Fannia canicularis). A description and analysis. J Comp Physiol 89: 331-357 
Laughlin SB (1994) Matching coding, circuits, cells, and molecules to signals: general principles of retinal design in the fly's eye. Prog Retinal Eye Research 13: 165-196

Laughlin SB, Howard J, Blakeslee B (1987) Synaptic limitations to contrast coding in the retina of the blowfly Calliphora. Proc Roy Soc Lond B 231: 437-467

Liebenthal E, Uhlmann O, Camhi JM (1994) Critical parameters of the spike trains in a cell assembly: coding of turn direction by giant interneurons of the cockroach. J Comp Physiol A 174: $281-296$

Lisberger SG, Movshon JA (1999) Visual motion analysis for pursuit eye movements in area MT of macaque monkeys. J Neurosci 19: 2224-2246

Maddess T, Laughlin SB (1985) Adaptation of the motion-sensitive neuron H1 is generated locally and governed by contrast frequency. Proc Roy Soc Lond B 225:251-275

Mainen ZF, Sejnowski TJ (1995) Reliability of spike timing in neocortical neurons. Science 268: 1503-1506

Mastebroek HAK (1974) Stochastic structure of neural activity in the visual system of the blowfly. Doctoral Dissertation, Rijksuniversiteit te Groningen

Mikami A, Newsome WT, Wurtz RH (1986) Motion selectivity in macaque visual cortex. II Spatiotemporal range of directional interactions in MT and V1. J Neurophysiol 55: 1328-1339

Miles FA, Wallman J (1993) Visual motion and its role in the stabilization of gaze. Elsevier, Amsterdam, London, New York

Movshon JA, Lisberger SG, Krauzlis RJ (1990) Visual cortical signals supporting smooth pursuit eye movements. Cold Spring Harb Symp Quant Biol 55: 707-716

Nowak LG, Sanchez-Vives MV, McCormick DA (1997) Influence of low and high frequency inputs on spike timing in visual cortical neurons. Cerebral Cortex 7: 487-501

O'Carroll DC, Bidwell NJ, Laughlin SB, Warrant EJ (1996) Insect motion detectors matched to visual ecology. Nature 382: 63-66

Reichardt W (1961) Autocorrelation, a principle for the evaluation of sensory information by the central nervous system. In: Rosenblith WA (ed) Sensory communication. MIT Press and John Wiley and Sons, New York, London, pp 303-317.

Reichardt W, Poggio T, Hausen K (1983) Figure-ground discrimination by relative movement in the visual system of the fly. Part II: Towards the neural circuitry. Biol Cybern 46 (Suppl): 1-30

Reichardt W, Poggio T (1976) Visual control of orientation behaviour in the fly. Part I. A quantitative analysis. Quart Rev Biophys 9: 311-375

Rieke F, Warland D, de Ruyter van Steveninck R, Bialek W (1997) Spikes. MIT Press, Cambridge, MA

Roberts A, Bush BMH (1981) Neurones without impulses. Cambridge University Press, Cambridge, London, New York

de Ruyter van Steveninck R, Bialek W (1988) Real-time performance of a movement-sensitive neuron in the blowfly visual system: Coding and information transfer in short spike sequences. Proc Roy Soc Lond B 234: 379-414

de Ruyter van Steveninck R, Bialek W (1995) Reliability and statistical efficiency of a blowfly movement-sensitive neuron. Phil Trans Roy Soc Lond B 348: 321-340

de Ruyter van Steveninck R, Laughlin SB (1996) The rate of information transfer at gradedpotential synapses. Nature 379: 642-645

de Ruyter van Steveninck R, Lewen GD, Strong SP, Koberle R, Bialek W (1997) Reproducibility and variability in neural spike trains. Science 275: 1805-1808

de Ruyter van Steveninck R, Zaagman WH, Mastebroek HAK (1986) Adaptation of transient responses of a movement-sensitive neuron in the visual system of the blowfly, Calliphora erythrocephala. Biol Cybern 54: 223-236

Schilstra C, van Hateren JH (1999) Blowfly flight and optic flow. I. Thorax kinematics and flight dynamics. J Exp Bio 202: 1481-1490

Shadlen MN, Britten KH, Newsome WT, Movshon JA (1996) A computational analysis of the relationship between neuronal and behavioral responses to visual motion. J Neurosci 16: 1486- 1510

Shadlen MN, Newsome WT (1998) The variable discharge of cortical neurons: implications for connectivity, computation, and information coding. J Neurosci 18: 3870-3896 
Shannon CE, Weaver W (1949) The mathematical theory of communication. The University of Illinois Press, Urbana

Stevens CF, Zador AM (1998) Input synchrony and the irregular firing of cortical neurons. Nature Neurosci 1: 210-217

Strausfeld NJ (1989) Beneath the compound eye: neuroanatomical analysis and physiological correlates in the study of insect vision. In: Stavenga DG, Hardie RC (eds) Facets of vision. Springer, Berlin, Heidelberg, New York, pp 317-359

Strong SP, Koberle R, de Ruyter van Steveninck R, Bialek W (1998) Entropy and information in neural spike trains. Physical Review Letters 80: 197-200

Theunissen F, Roddey JC, Stufflebeam S, Clague H, Miller JP (1996) Information theoretic analysis of dynamical encoding by four identified primary sensory interneurons in the cricket cercal system. J Neurophysiol 75: 1345-1364

Tolhurst DJ, Movshon JA, Dean AF (1983) The statistical reliability of signals in single neurons in cat and monkey visual cortex. Vis Res 23: 775-785

Virsik R, Reichardt W (1976) Detection and tracking of moving objects by the fly Musca domestica. Biol Cybern 23: 83-98

Vogels R, Spileers W, Orban GA (1989) The response variability of striate cortical neurons in the behaving monkey. Exp Brain Res 77: 432-436

Voss R, Zeil J (1998) Active vision in insects: An analysis of object-directed zig-zag flights in wasps (Odynerus spinipes, Eumenidae). J Comp Physiol A 182: 373-387

Wagner H (1986a) Flight performance and visual control of the flight of the free-flying housefly (Musca domestica). II. Pursuit of targets. Phil Trans Roy Soc Lond B 312: 553-579

Wagner H (1986b) Flight performance and visual control of flight of the free-flying housefly (Musca domestica). III. Interactions between angular movement induced by wide- and smallfield stimuli. Phil Trans Roy Soc Lond B 312: 581-595

Warzecha A-K (1994) Reliability of neuronal information processing in the motion pathway of the blowflies Calliphora erythrocephala and Lucilia cuprina. Doctoral Disseration, Universität Tübingen

Warzecha A-K, Egelhaaf M (1996) Intrinsic properties of biological motion detectors prevent the optomotor control system from getting unstable. Phil Trans Roy Soc Lond B 351: 1579-1591

Warzecha A-K, Egelhaaf M (1997) How reliably does a neuron in the visual motion pathway of the fly encode behaviourally relevant information? Europ J Neurosci 9: 1365-1374

Warzecha A-K, Egelhaaf M (1998) On the performance of biological movement detectors and ideal velocity sensors in the context of optomotor course stabilization. Vis Neurosci 15: 113-122

Warzecha A-K, Egelhaaf M. (1999) Variability in spike trains during constant and dynamic stimulation. Science 283: 1927-1930

Warzecha A-K, Kretzberg J, Egelhaaf M (1998) Temporal precision of encoding of motion information by visual interneurons. Curr Biol 8: 359-368

White JA, Rubinstein JT, Kay AR (2000) Channel noise in neurons. Trends Neurosci. 23: 131-137

Zanker JM, Egelhaaf M, Warzecha A-K (1991) On the coordination of motor output during visual flight control of flies. J Comp Physiol A 169: 127-134

Zeil J (1993) Orientation flights of solitary wasps (Cerceris, Sphecidae, Hymenoptera). I. Description of flights. J Comp Physiol 172: 189-205

Zohary E, Shadlen MN, Newsome WT (1994) Correlated neuronal discharge rate and its implications for psychophysical performance. Nature 370: 140-143

\section{Note added in proof}

In this volume de Ruyter van Steveninck et al. argue that we reached "conclusions so nearly opposite" ( $p$ 303) from their own. They come to this view by attributing conclusions to us that represent caricatures of the conclusions we have drawn. Moreover, they claim that our analysis has severe theoretical shortcomings. This 
claim is unwarranted and results from misinterpreting our data analysis (compare their pp 290-293 with our pp 251-257 and their pp 293-298 with our pp 269-272). There are no principal differences in the experimental data obtained by both groups but merely discrepancies concerning interpretations. Whereas de Ruyter van Steveninck et al. conclude that "individual spikes are reproducible on a millisecond time scale" (p 303), we argue that in motion-sensitive neurones only part of the spikes elicited under natural conditions are timed as precisely: The precision of spike timing is determined by the membrane potential changes at the spike initiation zone and the dynamics of these membrane potential changes depends on the dynamics of the visual input. At least if we accept that under natural conditions visual motion is not only the consequence of saccade-like turns of the animal (see our pp 261-266), spikes inevitably lock to visual motion stimuli on a wide range of timescales. 